Journal of Social Sciences (COES\&RJ-JSS)

ISSN (E): 2305-9249 ISSN (P): 2305-9494

Publisher: Centre of Excellence for Scientific \& Research Journalism, COES\&RJ LLC

Online Publication Date: $1^{\text {st }}$ October 2017

Online Issue: Volume 6, Number 4, October 2017

https://doi.org/10.25255/jss.2017.6.4.833.850

\title{
Knowledge Management and its Role on Organizational Crisis Management: A Literature Review
}

\author{
Abdullatef Alkandari*, Dr. Ra'ed Masa'deh**, Prof. Musa Al-Lozi***
}

\begin{abstract}
:
This research aimed to study the concept of knowledge, knowledge management and its role on organizational crisis management through conducting a comprehensive review of theories, literature, and empirical studies. Furthermore, the researchers reviewed the prevailing theories of knowledge management and its relationship with organizational crisis management.
\end{abstract}

\section{Keywords:}

Knowledge management, organizational emergency management.

\section{Citation:}

Alkandari, Abdullatef; Masa'deh, Ra'ed; Al-Lozi, Musa (2017); Knowledge management and its role on organizational crisis management: a literature review; Journal of Social Sciences (COES\&RJ-JSS), https://doi.org/10.25255/jss.2017.6.4.833.850.

* M.S.c. Candidate at the University of Jordan Business School, Amman, Jordan, ** Associate Professor of Management Information Systems Department, The School of Business, The University of Jordan,

*** Vice President for Centers and Community Service Affairs, President of Aqaba Branch, The University of Jordan, 


\title{
Knowledge management and its role on organizational ....
}

\author{
إدارة المعرفة ودور ها على إدارة الأزمات التنظيمية: دراسة نظرية \\ عبداللطيف الكندري 1 ، د.رائد (محمد تيسير) مساعده 2، أ.د. موسى اللوزي 3

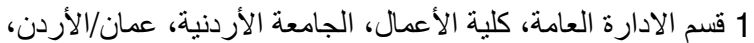

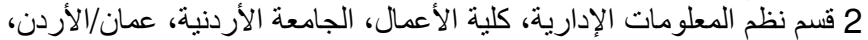

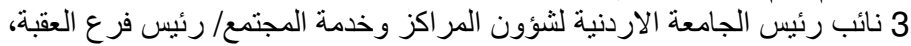

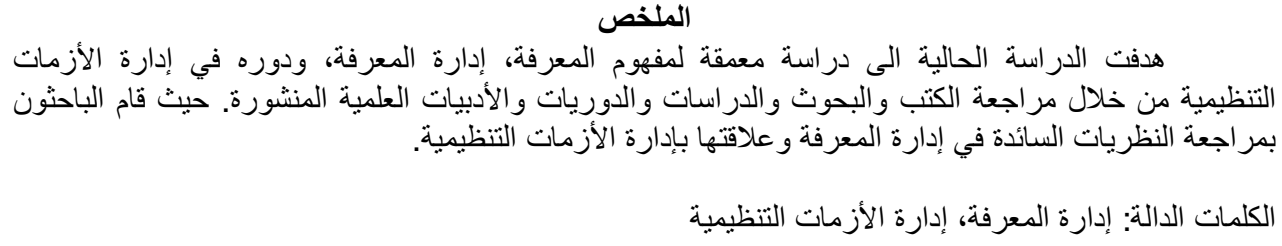

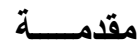

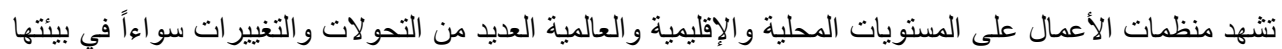

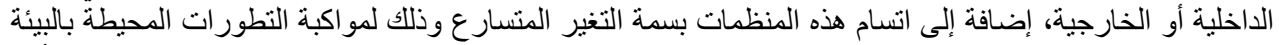

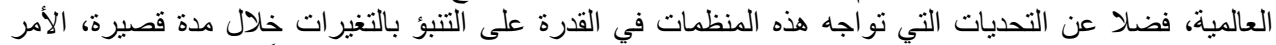

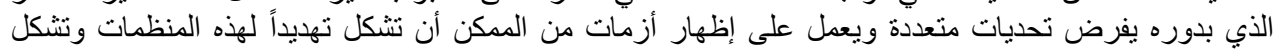

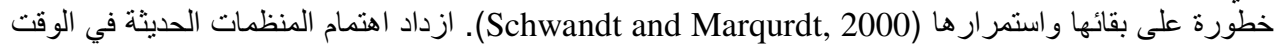

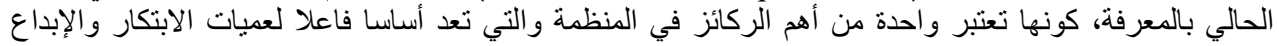

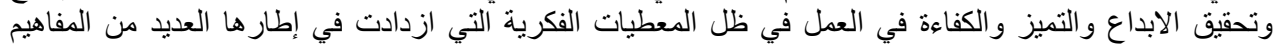

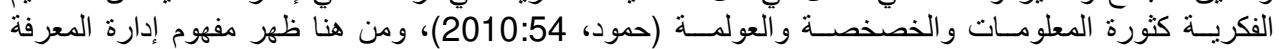

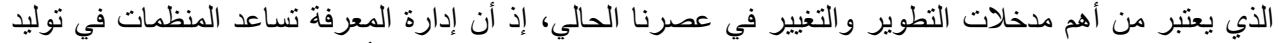

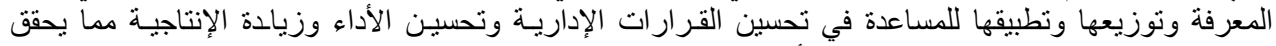

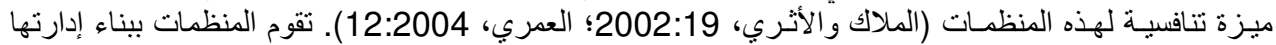

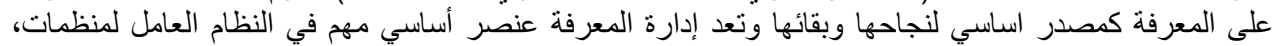

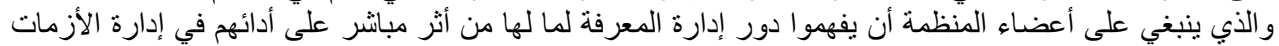

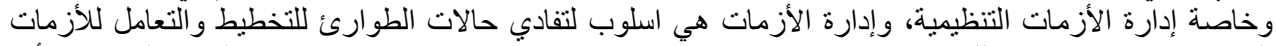

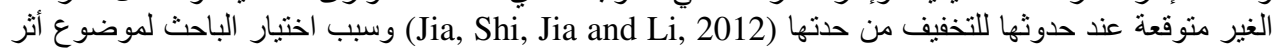

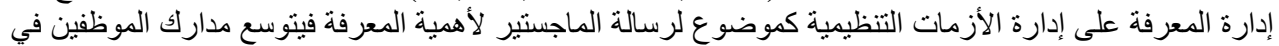

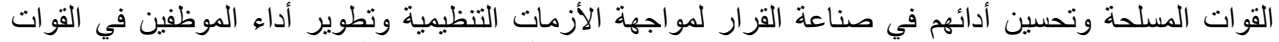
المسلحة يتطلب بناء معرفة تسمح بإجر اء تغير ات اليجابية لمو اجهة الأزمات التي تنشأ في المنظمات التهات

أولا: المعرفة المبنة

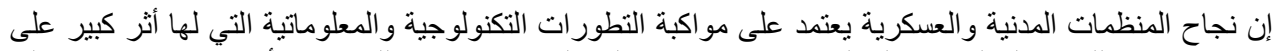

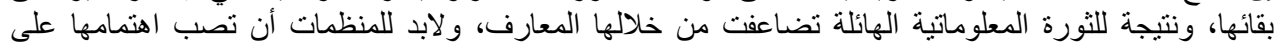

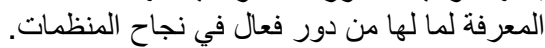

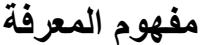

أسهم الكثير من الباحثين بإبداء آرائهم حول صياغة مفاهيم معينة للمعرفة، فقد عرفها قاموس (Oxford,1994) بأنها

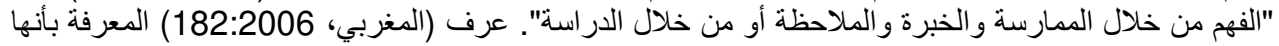

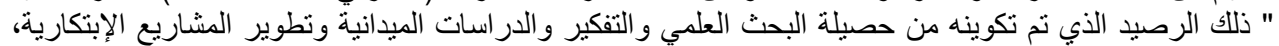

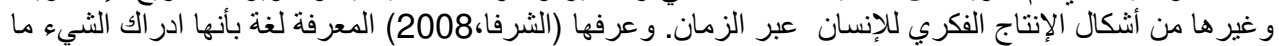

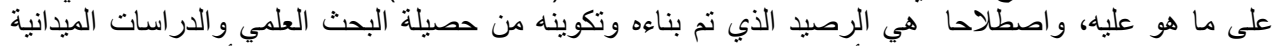

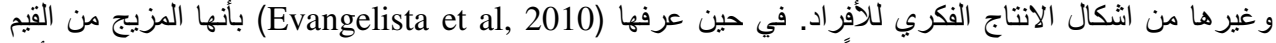

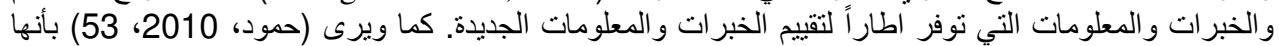

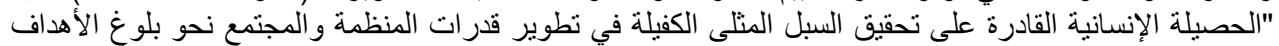

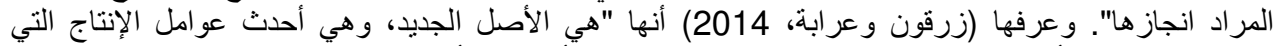

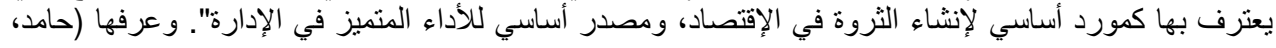




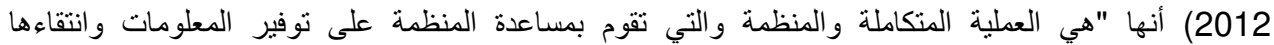

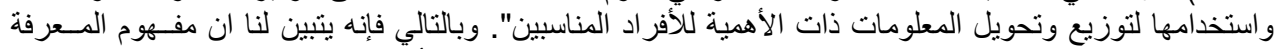

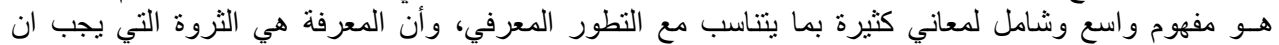

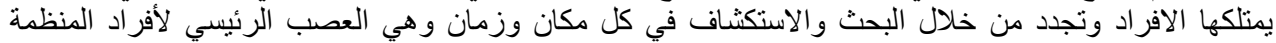

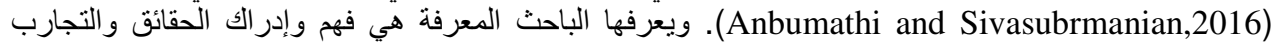

$$
\text { والمواقف التي يمر بها الفرد في حياته اليومية. }
$$

مصنادر المعرفية الميرة

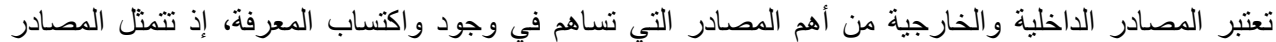

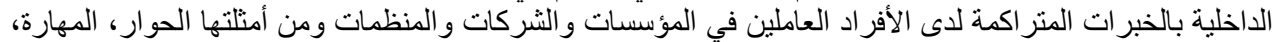

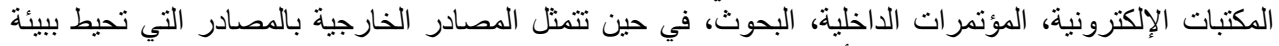

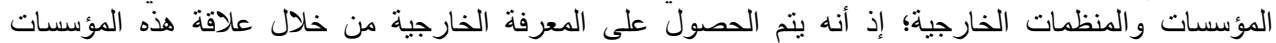

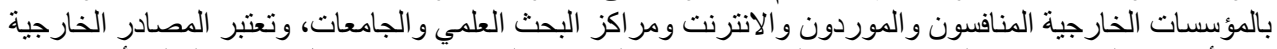

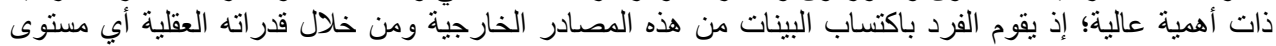

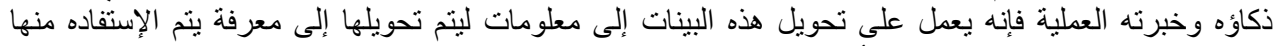
Altamony et al., 2012; Masa'deh and ) كما أن بعض الباحثين (Peacock, et al, 2015) Shannak, 2012; Masa'deh, 2012, 2013; Shannak et al., 2010; Masa'deh et al., 2008, 2013, 2014, 2015, 2017; Hajir et al., 2015; Kateb et al., 2015; Obeidat et al.,

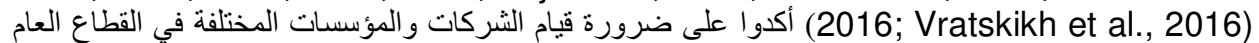

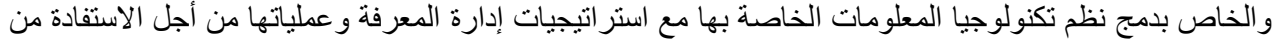

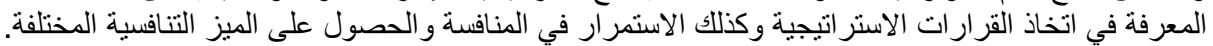

عناصر المعرفة في أخدان

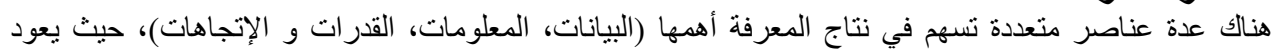

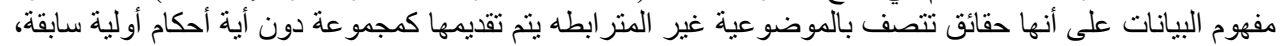

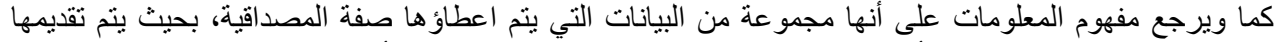

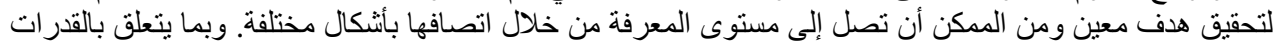

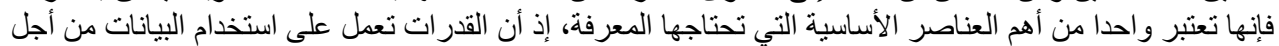

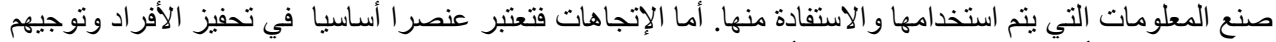

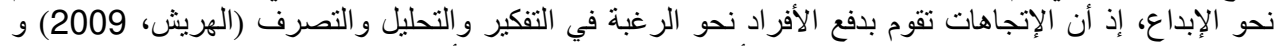

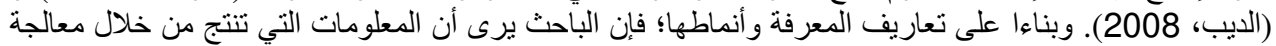

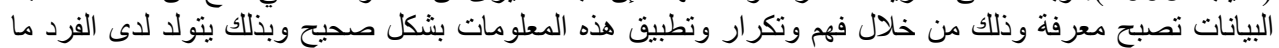

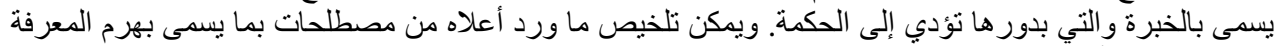

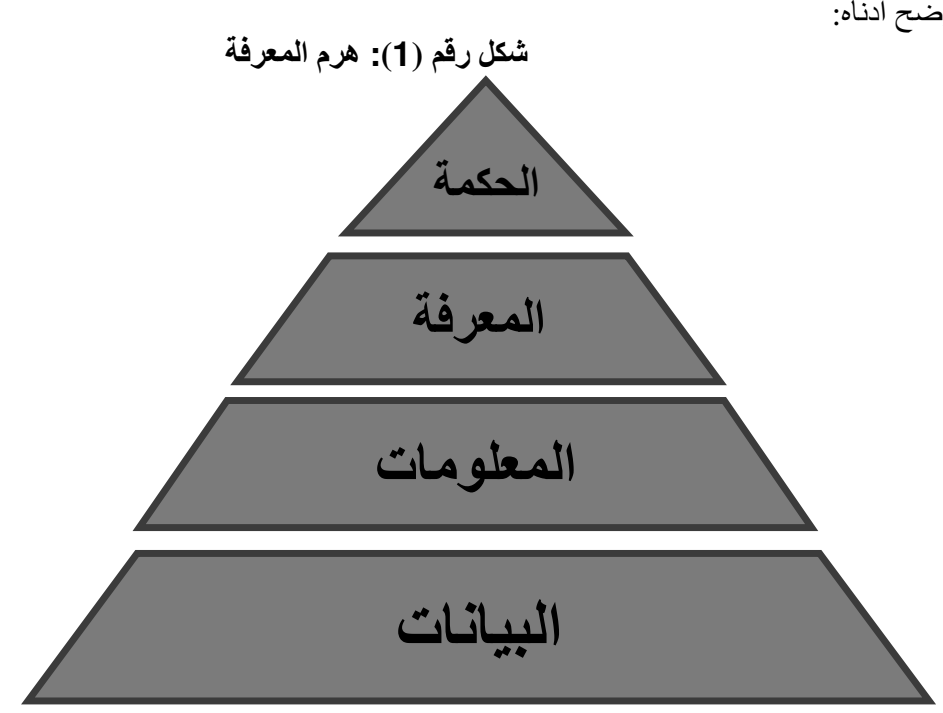

المصدر: همشري، عمر (2013)، إدارة المعرفة الطريق إلى التميز والريادة. 


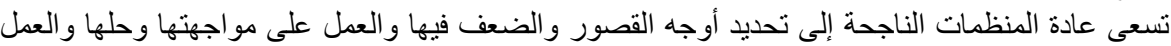

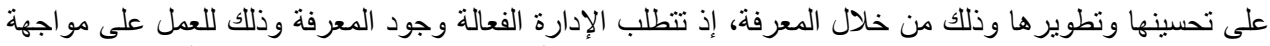

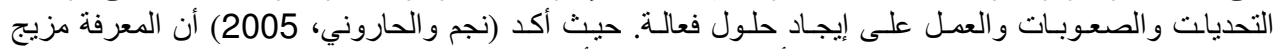

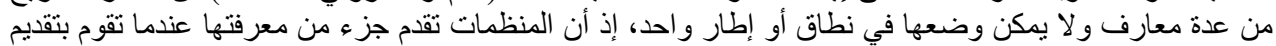

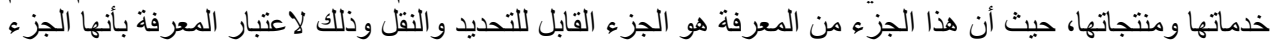

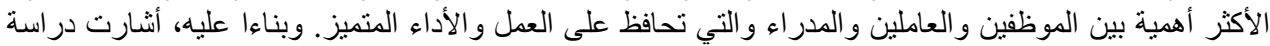

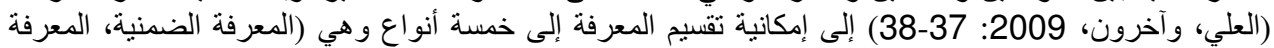

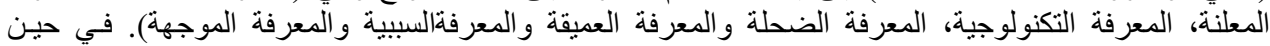

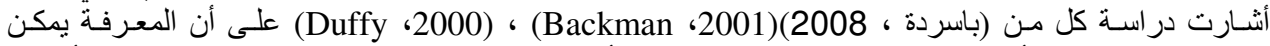

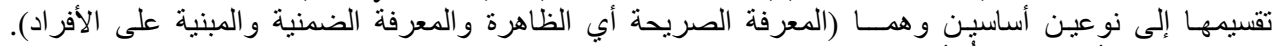
ويوضح الثكل أدنها ملخصاُ لأنماط المعرفة كما يلي:

شكل رقم (2): أنماط المعرفة

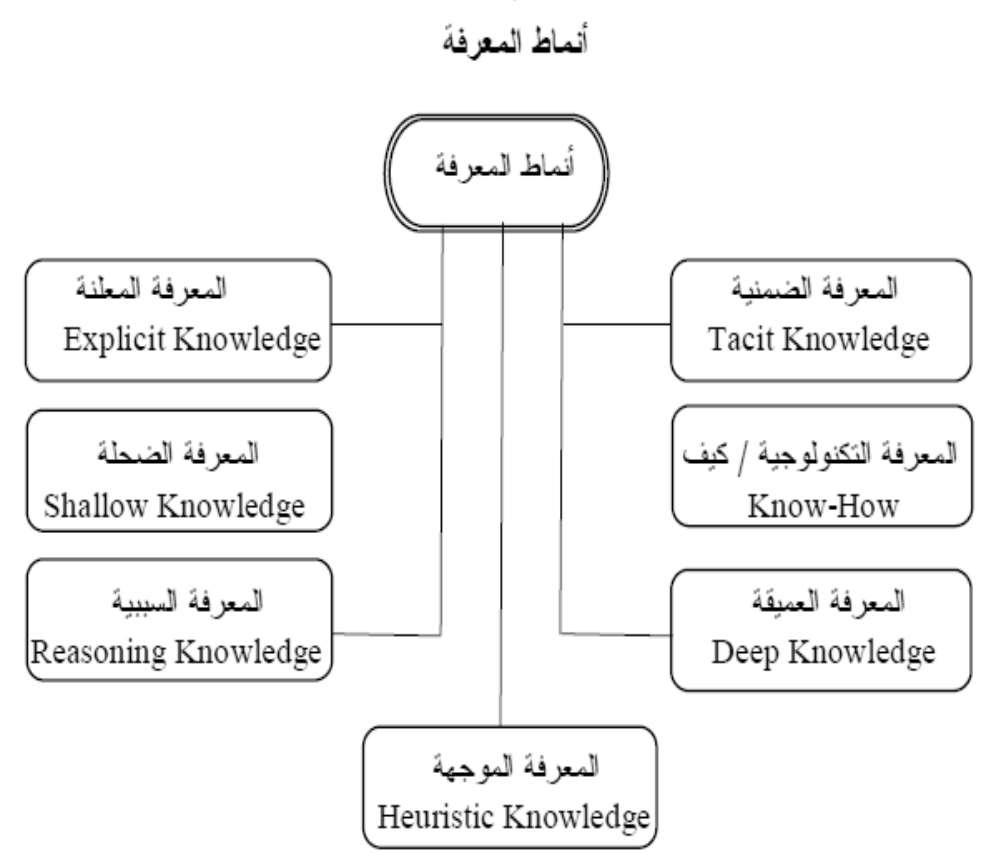

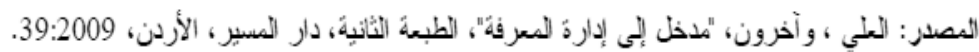

وقد ذكر (Ndlela, 2012) بوجود عو امل ضمنية تؤثر بشكل مباشر على تبادل المعرفة لإدارة الأزمات

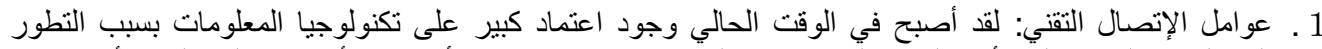

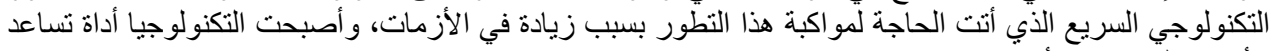
الأفر اد على إدارة الأزمات الإن.

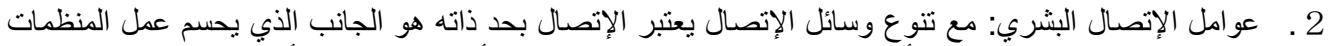

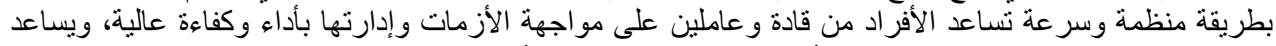

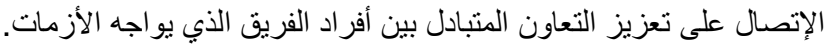


3 ـ هيكل الثبكة: إن شبكات إدارة الأزمات ماهي إلا هياكل معقدة من الترابط وتعمل هذه الثبكات على استر اتيجيات

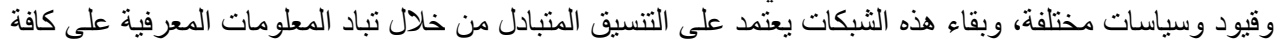
المستويات التنظيمية داخل المنظمة.

وقد قال (Ismail, 2015) أن المعرفة الضمنية هي المعرفة الثخصية التي تحدد السياق الذي من الصعب إنباء إضفاء

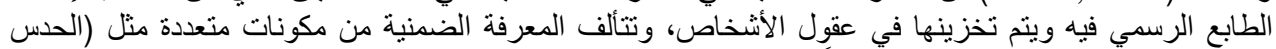

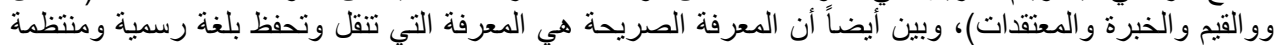

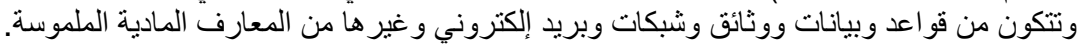

\section{ثانيا: - إدارة المعرفة

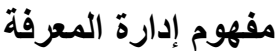

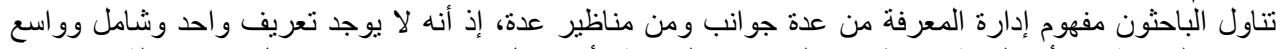

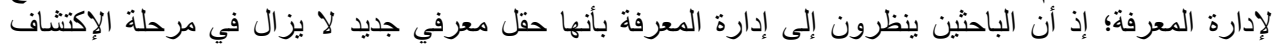

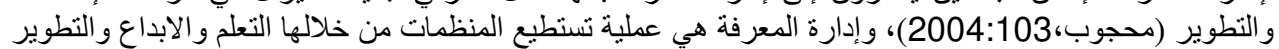

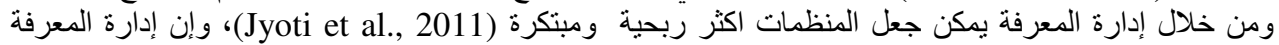

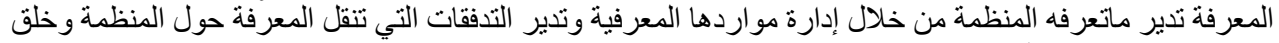

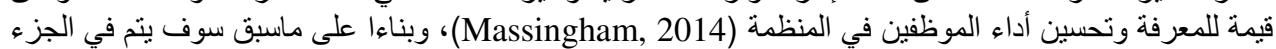

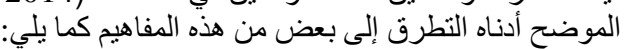

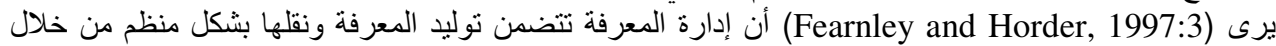

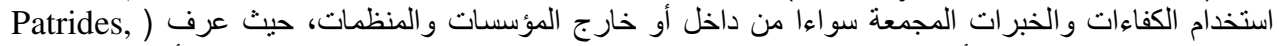

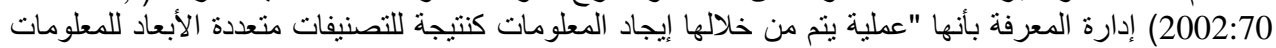

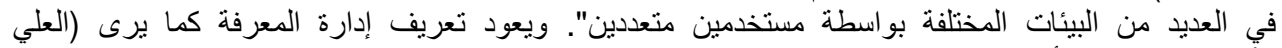

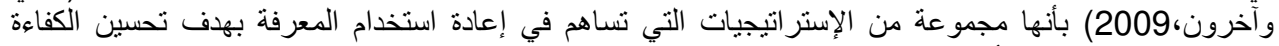

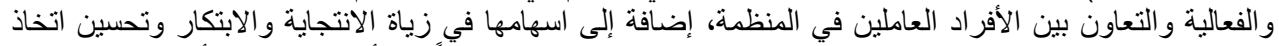

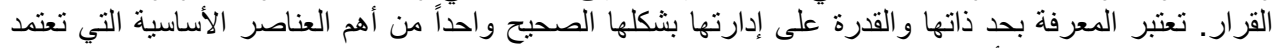

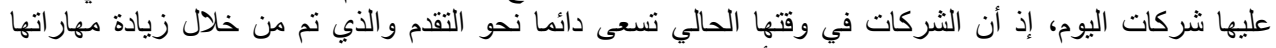

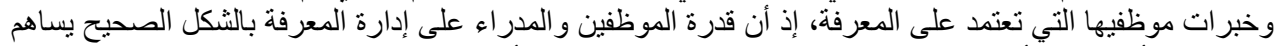

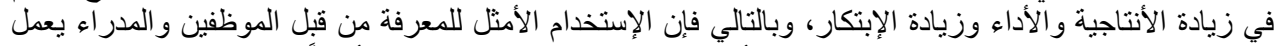

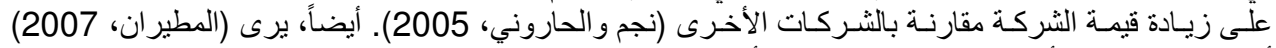

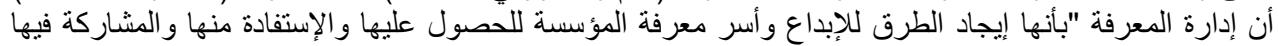

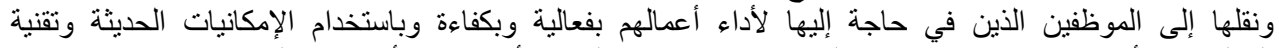

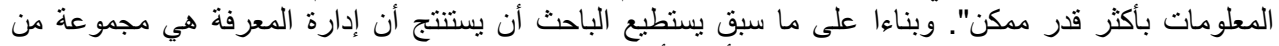

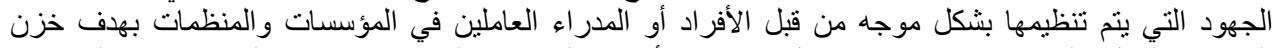

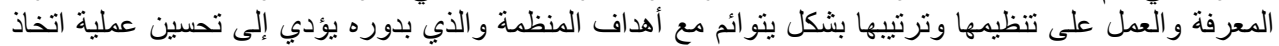

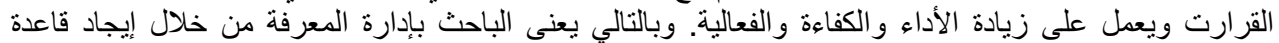

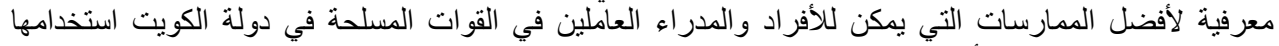
بهذف التقليل من مخاطر الأزمات الثمات التنظيمية.

وظائف إدارة المعرفة من ماطية

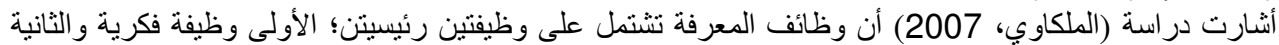

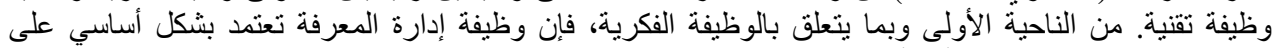

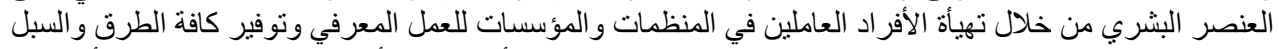

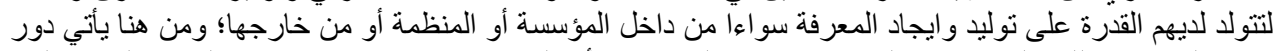

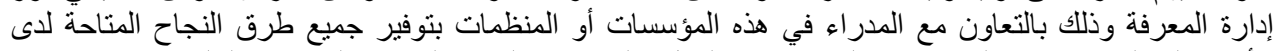

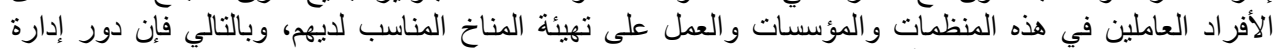

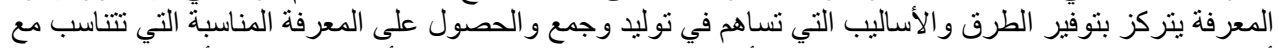

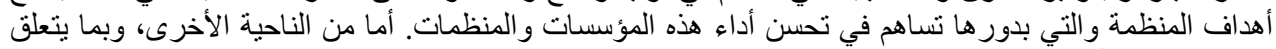

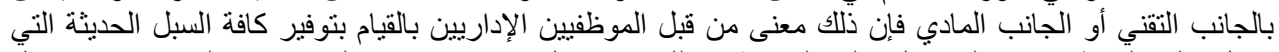

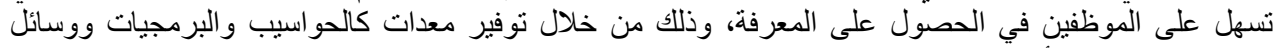

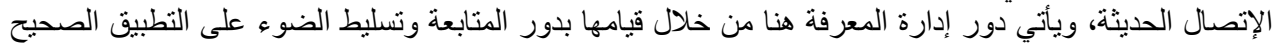

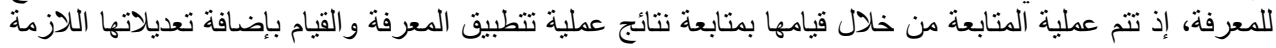




\section{Knowledge management and its role on organizational ....}

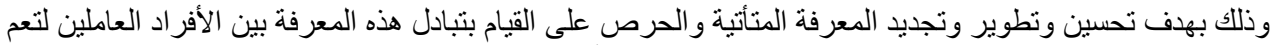

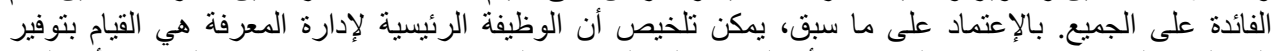

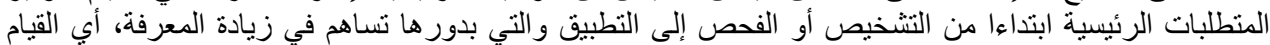

بتوفير كافة الطرق أو الوسائل التي تؤدي إلى المعرفة التئ.

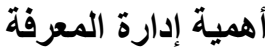

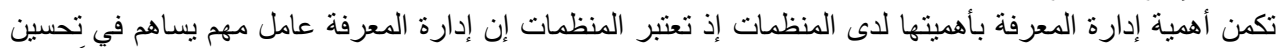

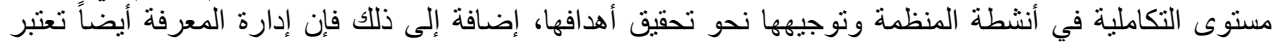

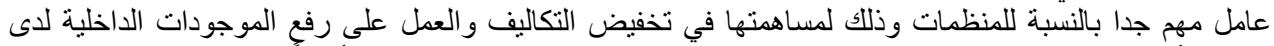

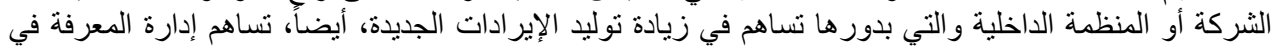

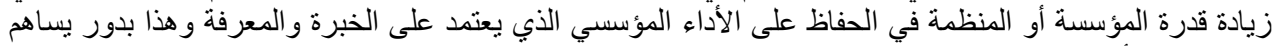

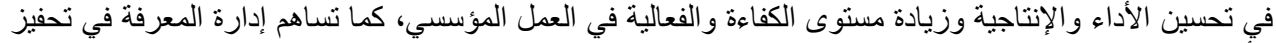

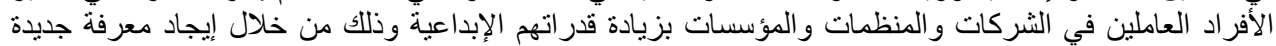

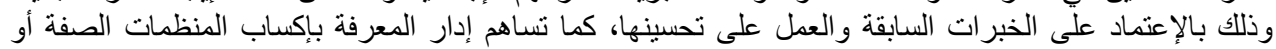

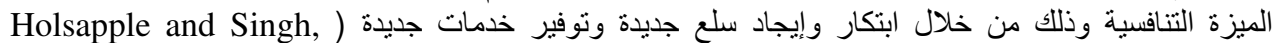

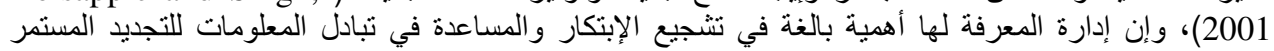

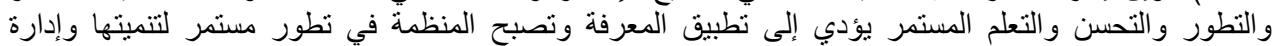

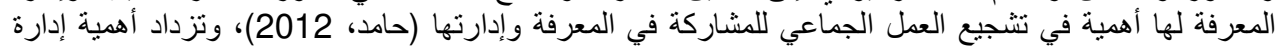

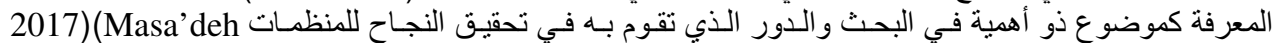
(et al,

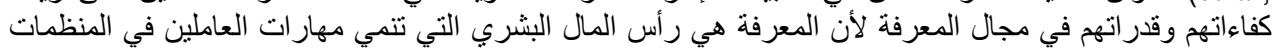

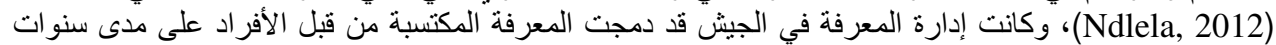

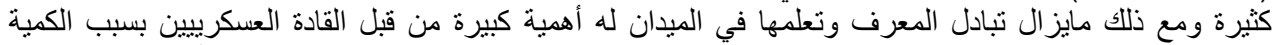

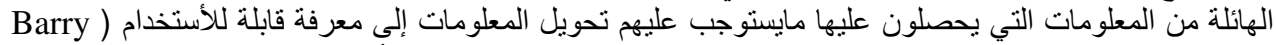

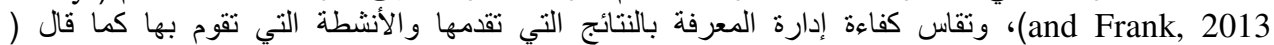

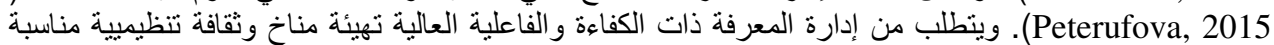

2.

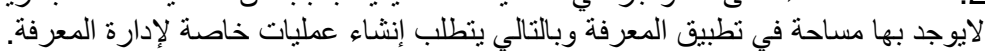

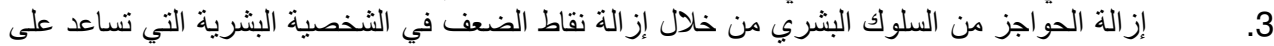
4ملية التعاون المنبادل فيما بينهم ونقل المعرفة.

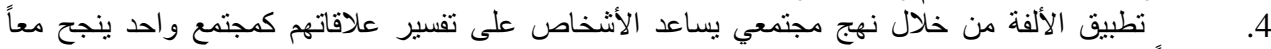
ويفشل معاً ويخلق قيم مشتركة فيما بينهم.

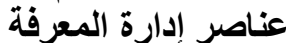

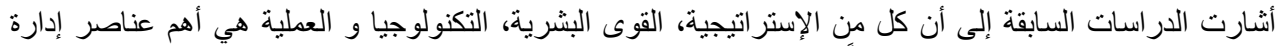

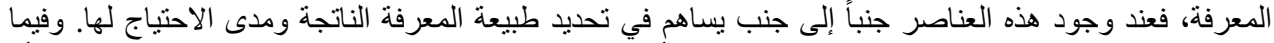

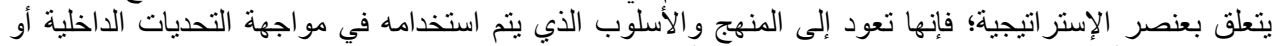

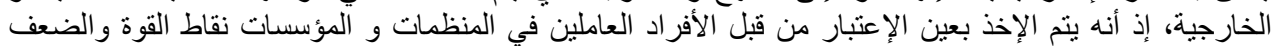

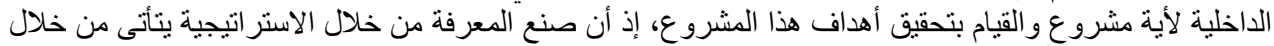

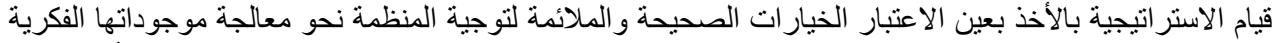

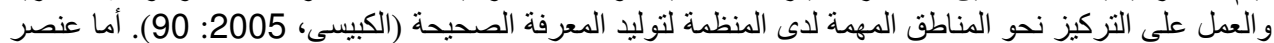

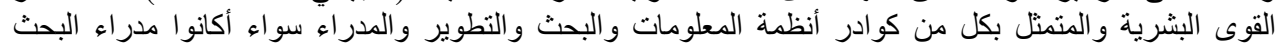

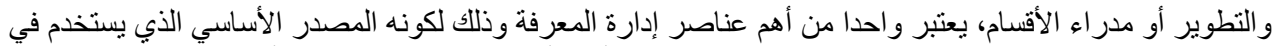

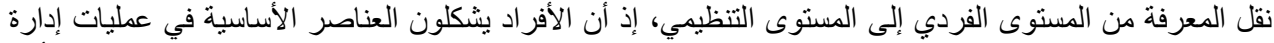

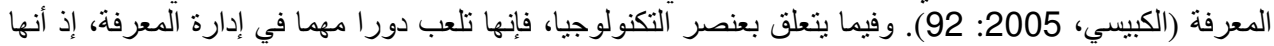

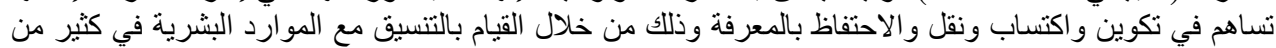

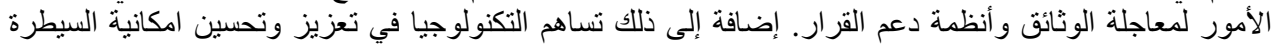

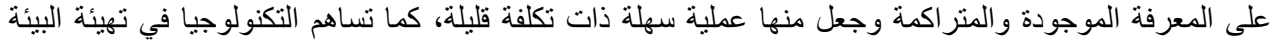

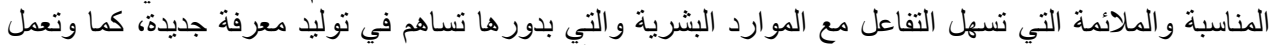

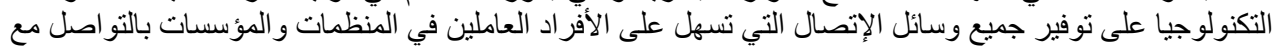




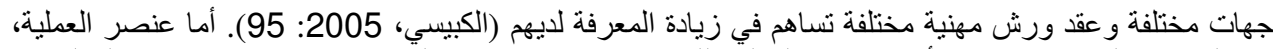

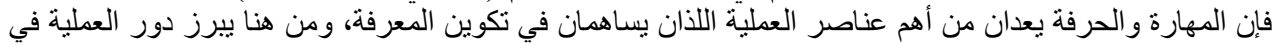

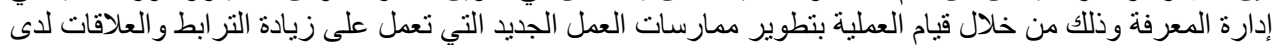

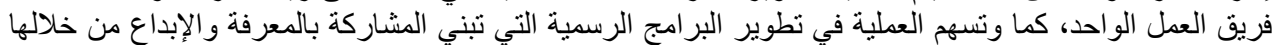

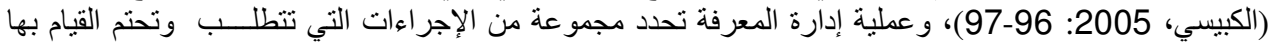

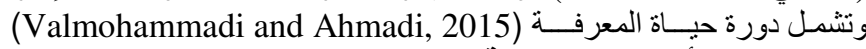
ثالثا: إدارة الأزمات التنظيمية

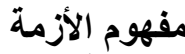
تعرف الأزمة (Crisis) الرطلاحا على أنها "الثدة والقحط، وأزم عن الثيء أي أمسك عنه، والأزمة الحمية، والمأزم

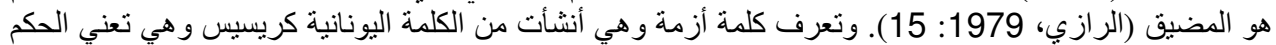

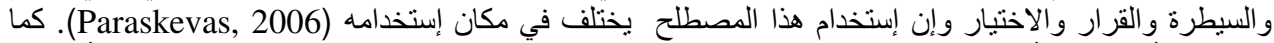

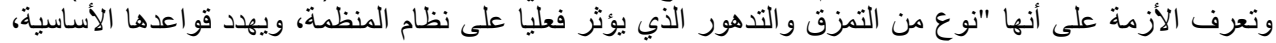

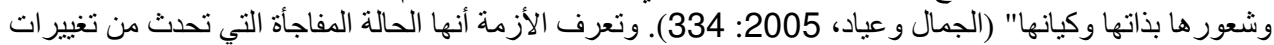

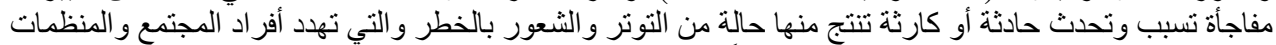

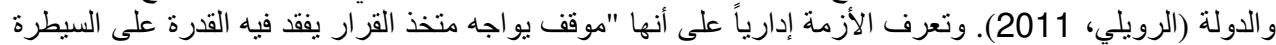

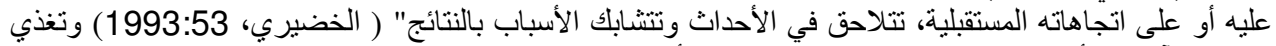

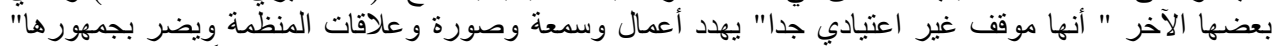

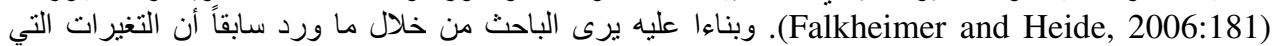

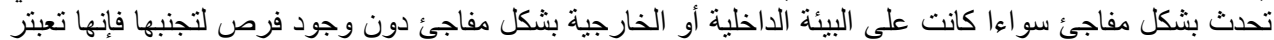

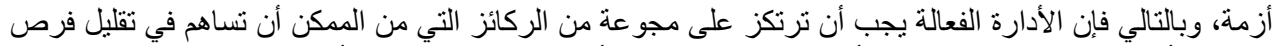

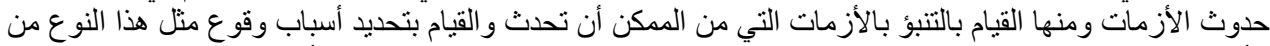

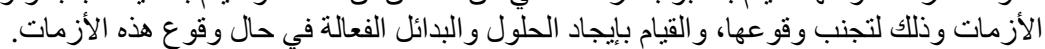

أسباب الأزمات ألات

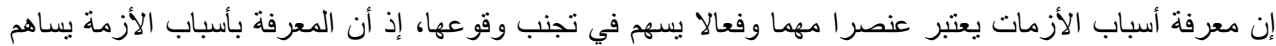

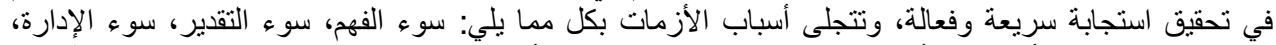

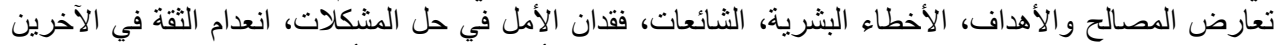

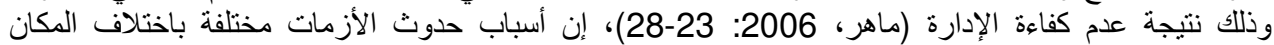

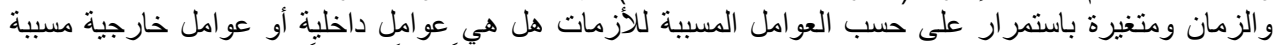

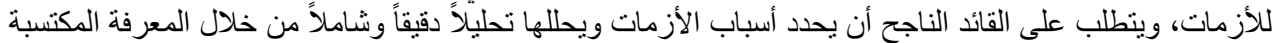

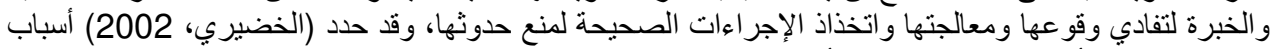
مختلفة لحدوث الأزمات كما هو موضح أدناه في الخير 
شكل رقم (3): أسباب نثوء الأزمات

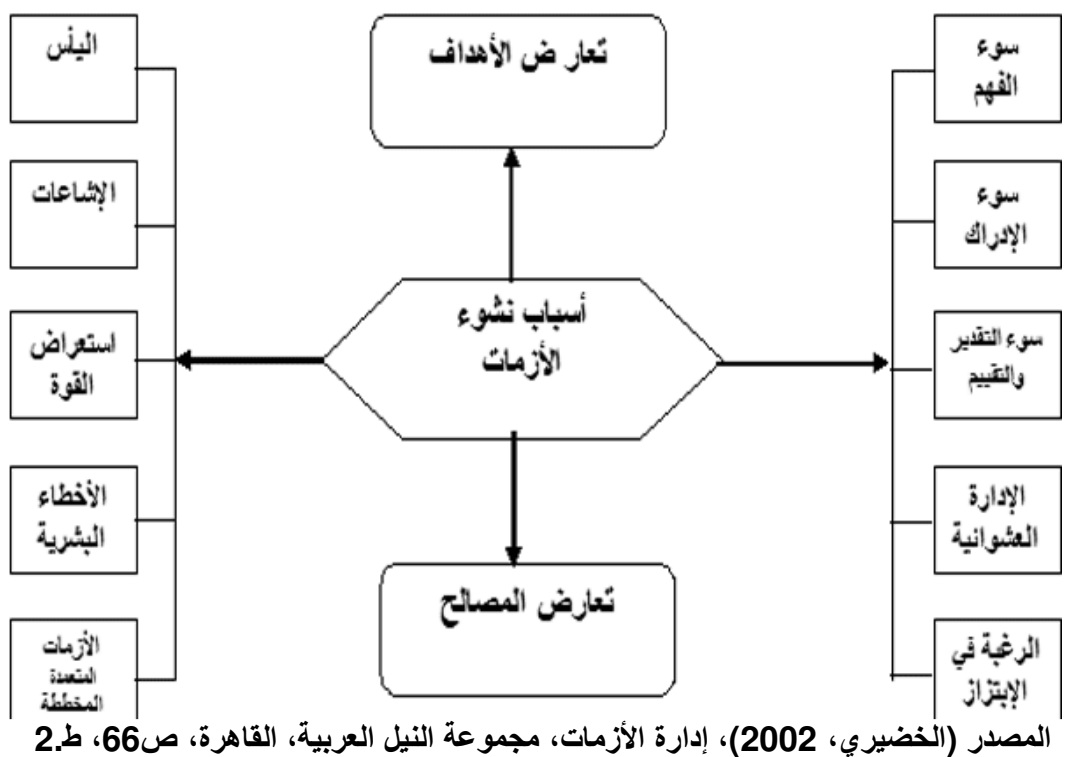

وقد ذكر (Hutchins and Wang, 2008) في دراسته أنه هنالك خمسة عوامل مترابطة تؤثر على أحداث إدارة

الأزمات في المنظمات وهي:

1.

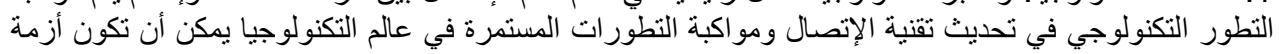
كبرى تهدد بقاء المنظمة.

2.

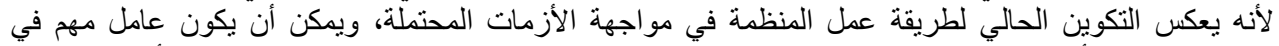

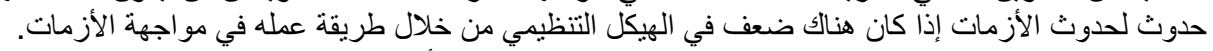

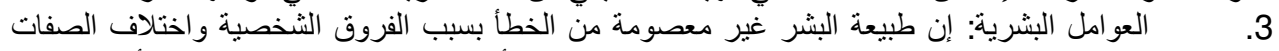

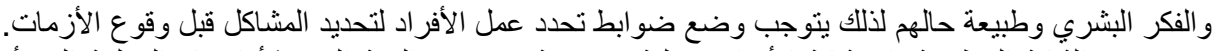

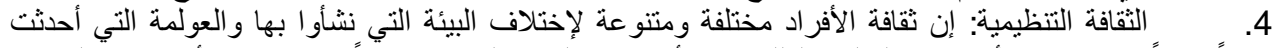

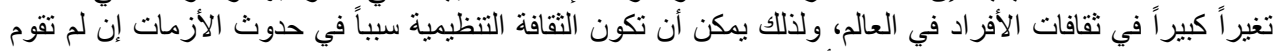

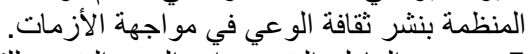

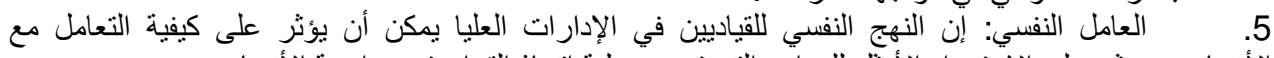
الأزمات، ويؤثر على الإستخدام الأمثل للموارد التي تدعم عملية اتخاذ القرار في مواتيات الفهة الأزمات.

أنواع الأزمات

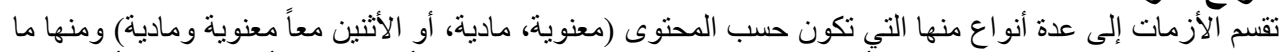

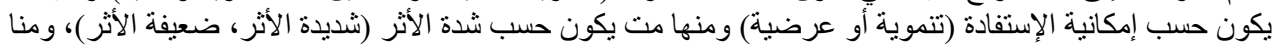

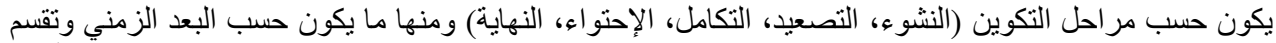

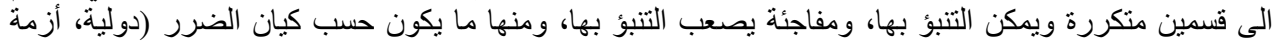

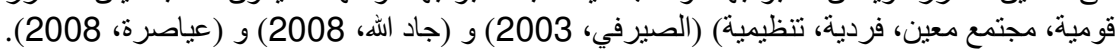

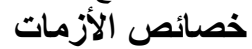

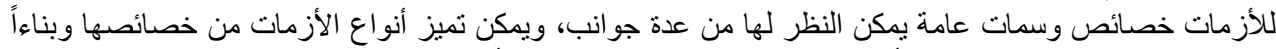

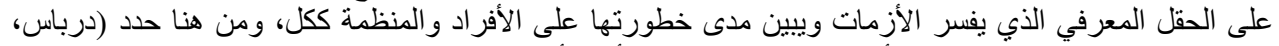

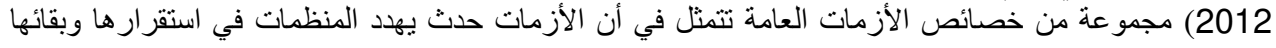




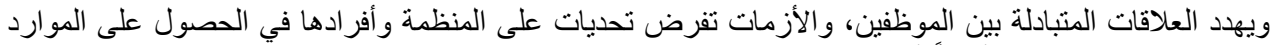

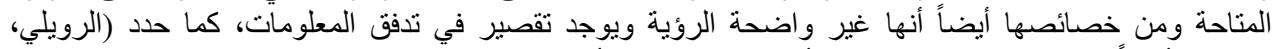

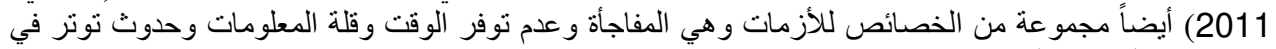

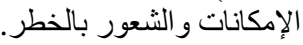

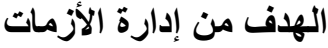

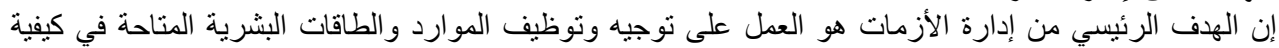

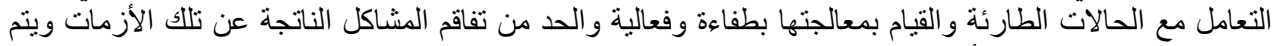

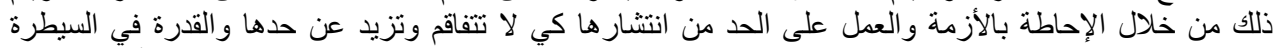

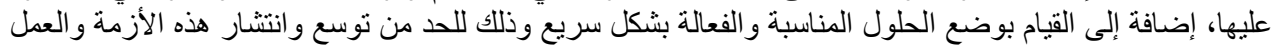

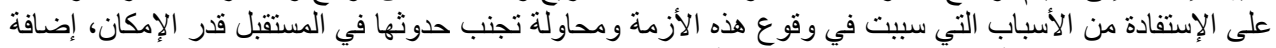

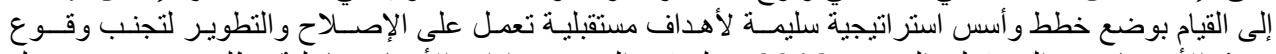

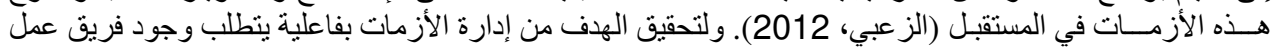

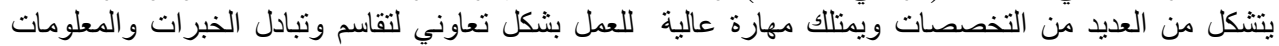
والأفكار التي تساعد أفراد الفريق في مواجهة الأزمات بالمعرفة المتبادلة من الفريق (Ndlela, 2012).

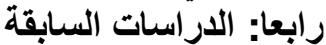

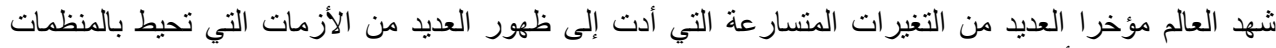

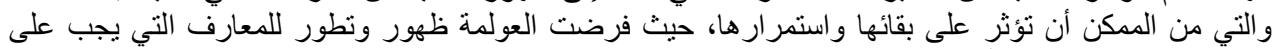

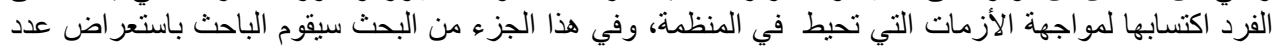

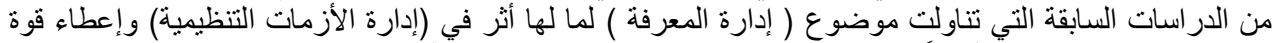

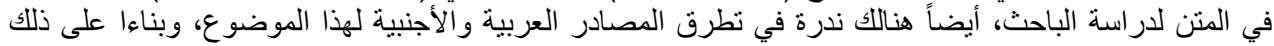

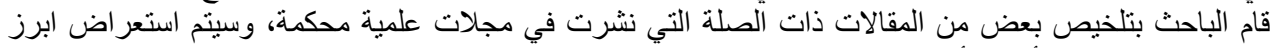

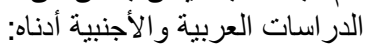

دراسة أبو عمر (2009)، فاعلية نظم المعلومات الادارية المحوسبة وأثرها على إدارة الأزمات:هدفت

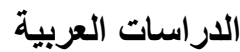

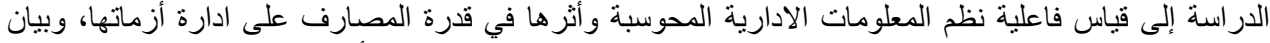

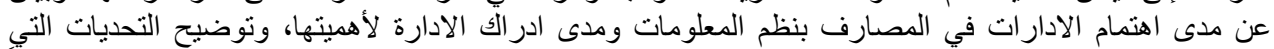

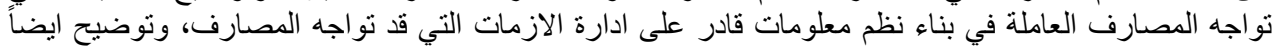

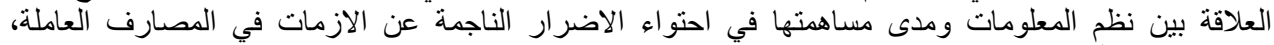

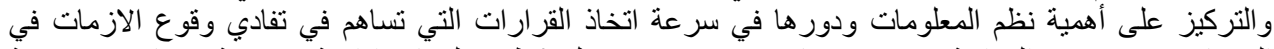

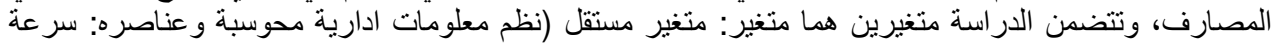

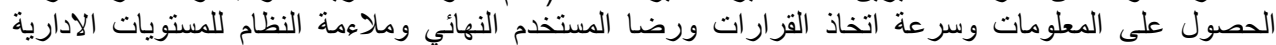

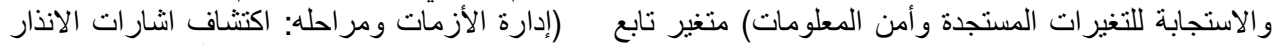

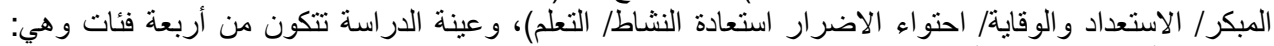

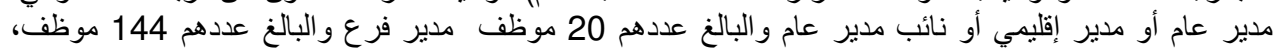

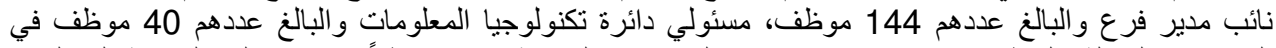

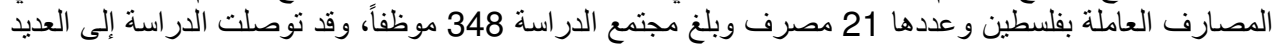

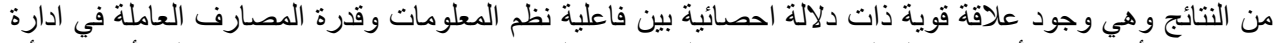

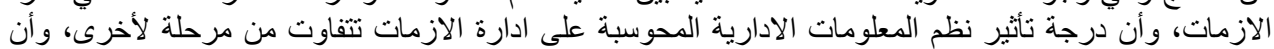

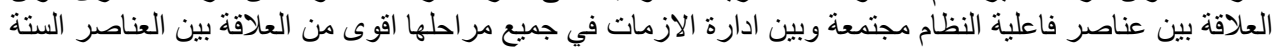

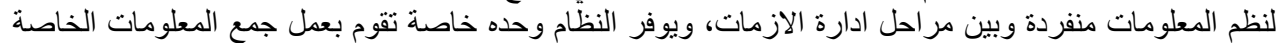
بالأزمات وتحليلها وتخزينها على قو اعد بيانات.

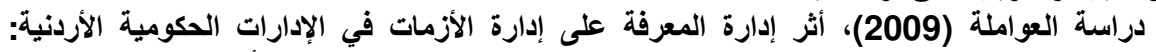

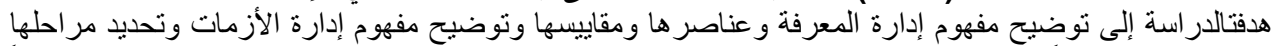

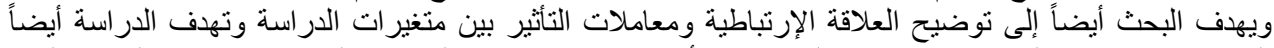

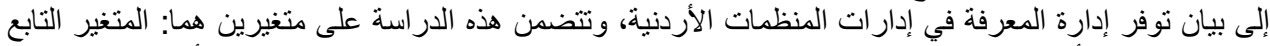

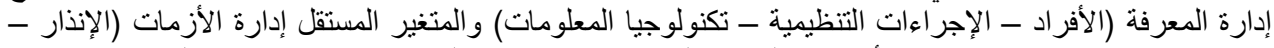

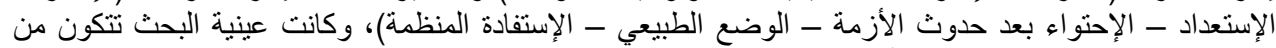

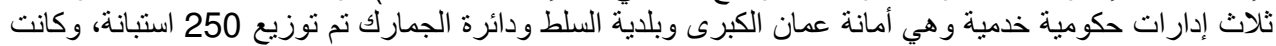

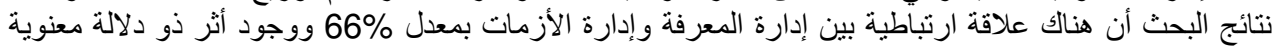




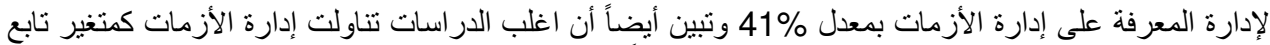

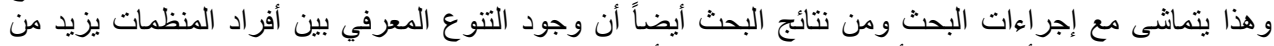

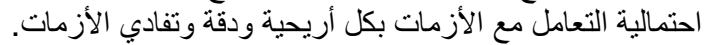

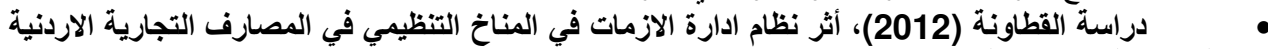

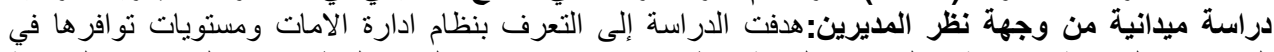

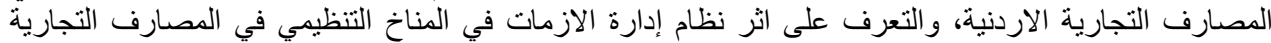

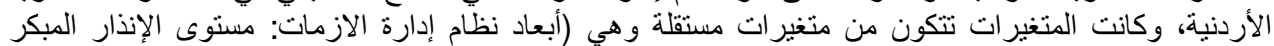

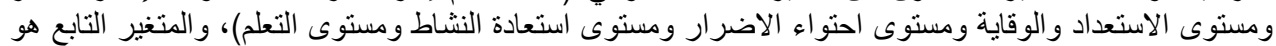

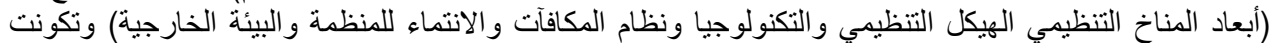

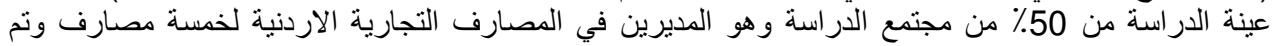

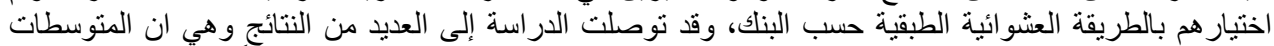

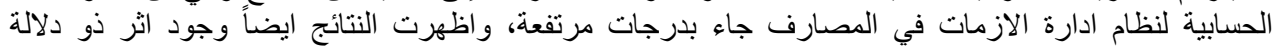

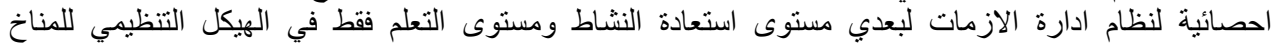

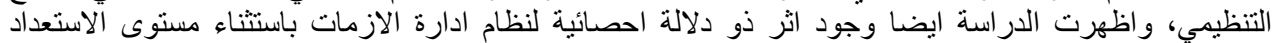

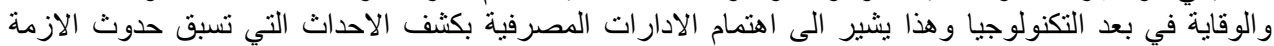

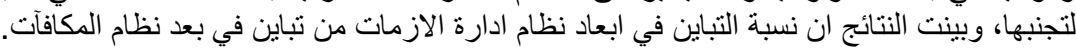

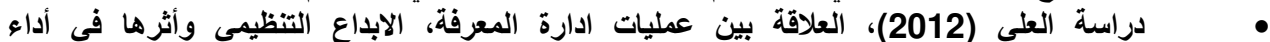

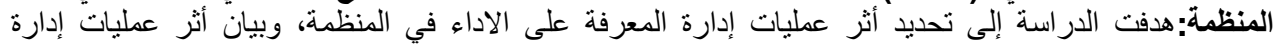

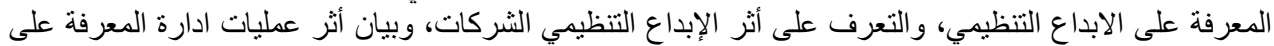

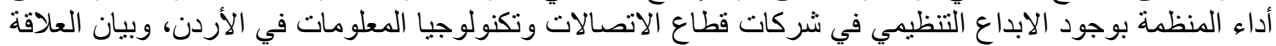

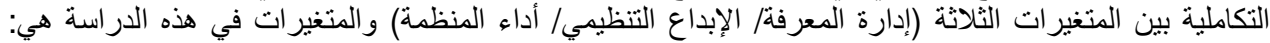

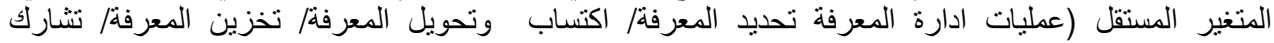

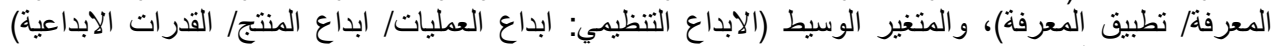

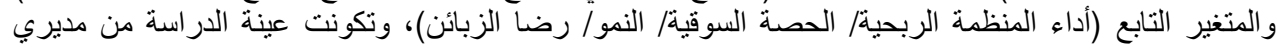

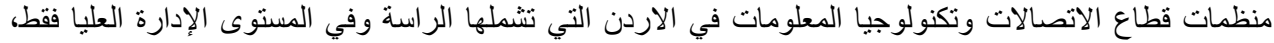

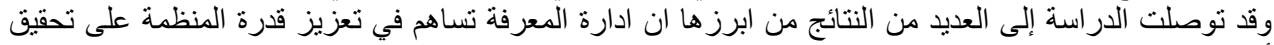

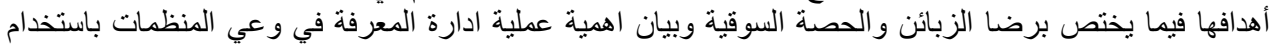

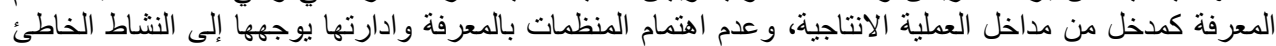

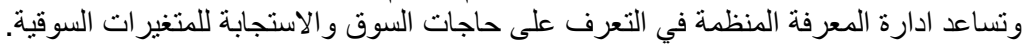

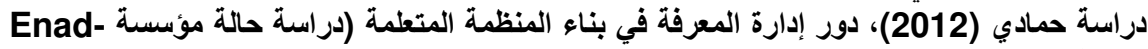

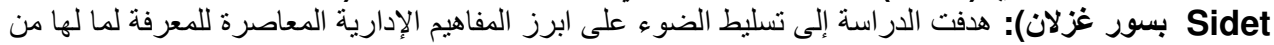

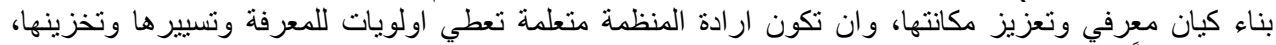

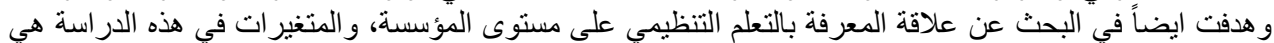

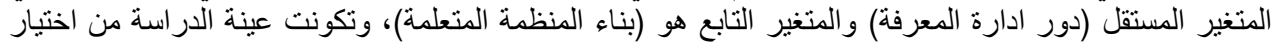

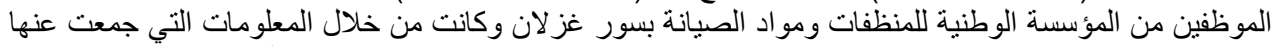

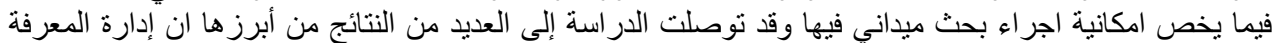

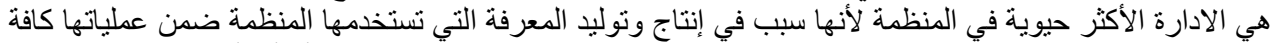

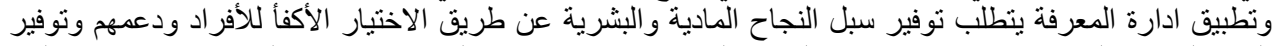

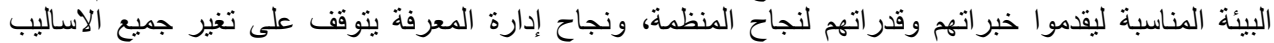

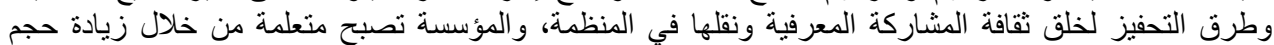

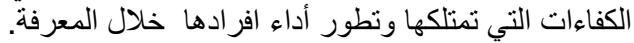

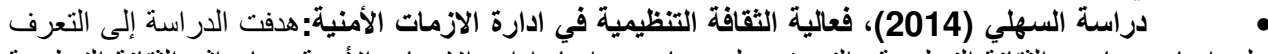

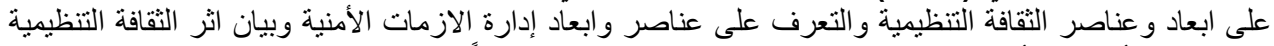

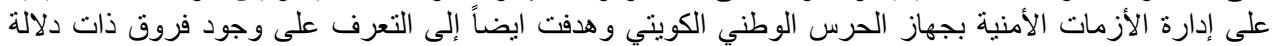

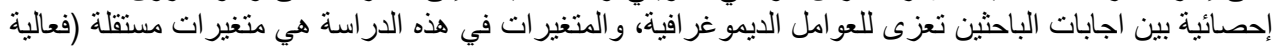

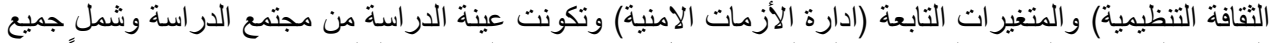

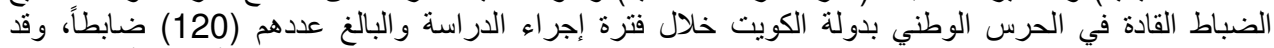

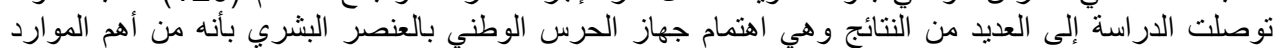

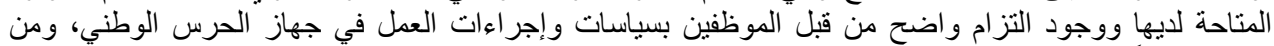

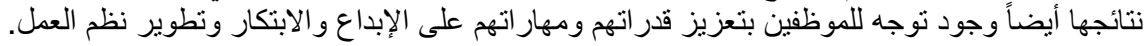




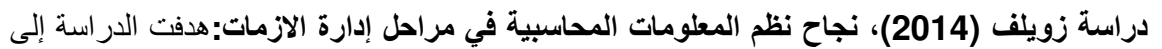

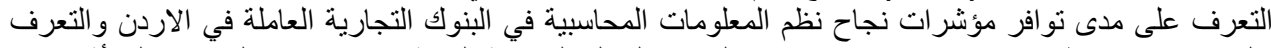

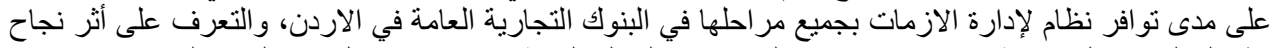

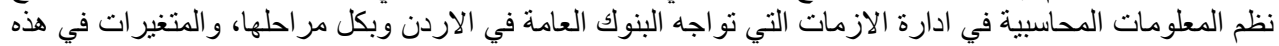

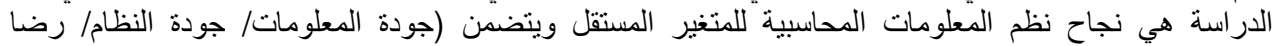

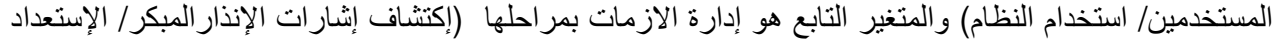

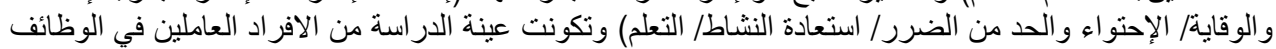

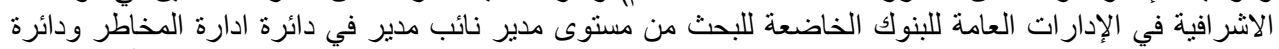

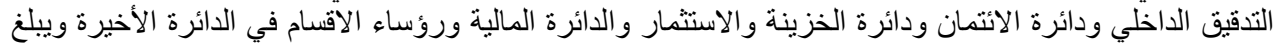

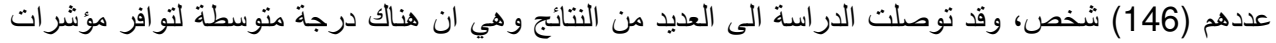

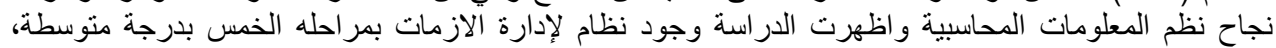

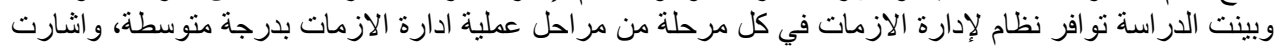

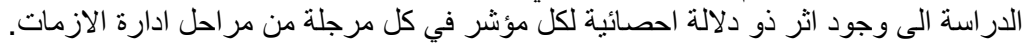

•

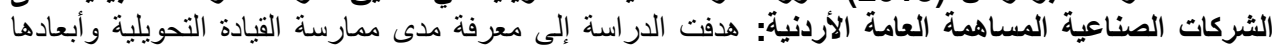

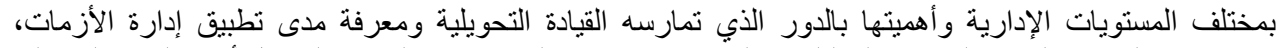

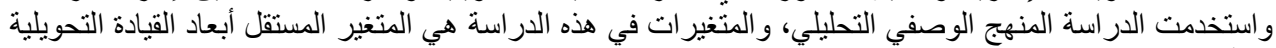

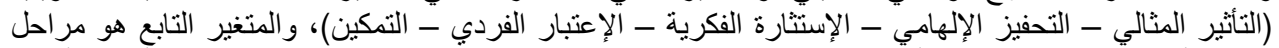

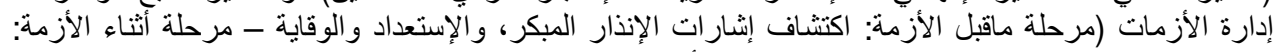

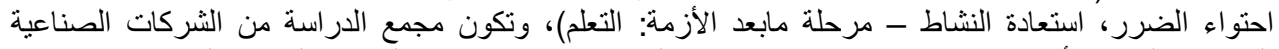

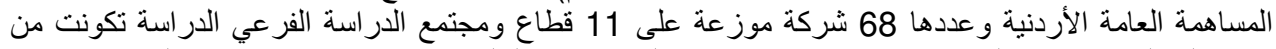

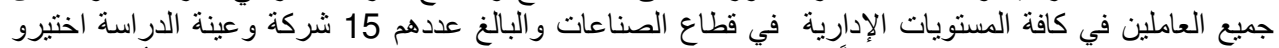

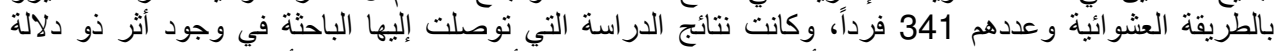

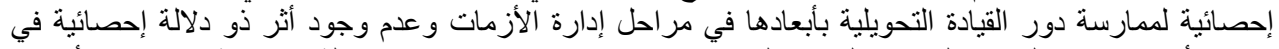

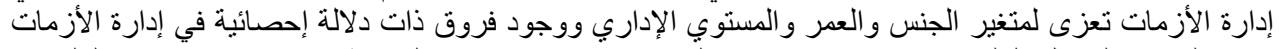

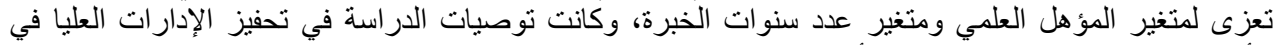
الأخذ بالإعتبار نمط القيادة التحويلية العيلية بأبعادها.

الاراسات الأجنبية

دراسة ( Shaw, et al., ( 2007 الاستجابة إلى الأزمة من خلال إدارة المعرفة الاستر اتيجية: هدفت

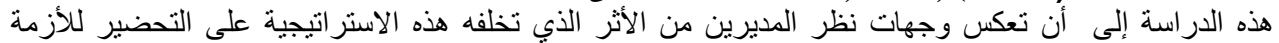

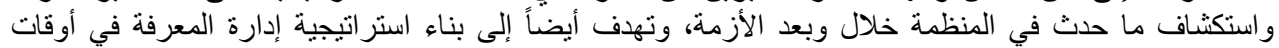

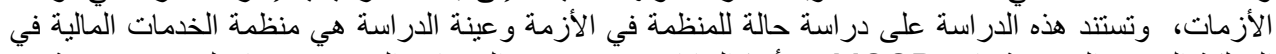

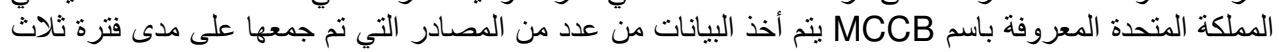

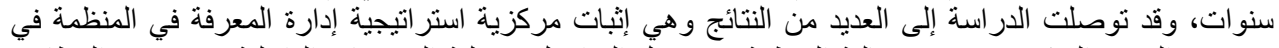

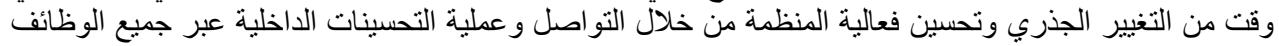
وخاصة بين خدمات التسجيل وفريق الامثكال.

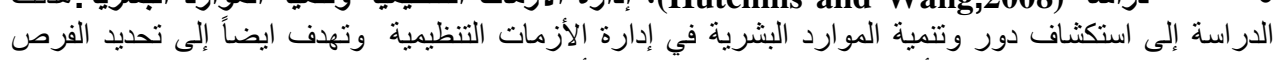

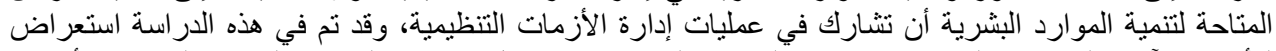

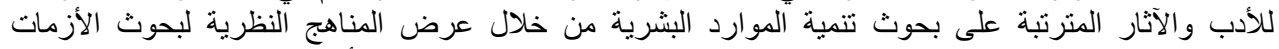

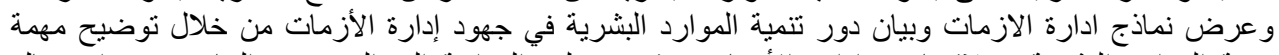

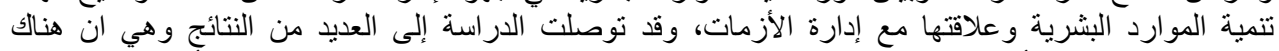

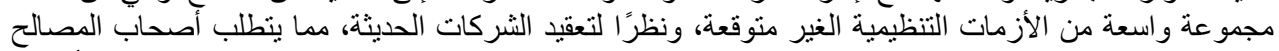

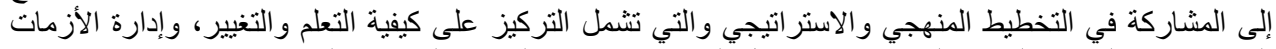

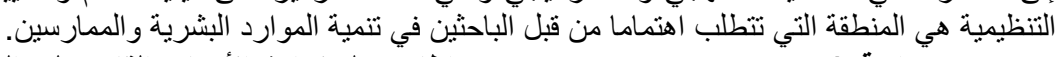

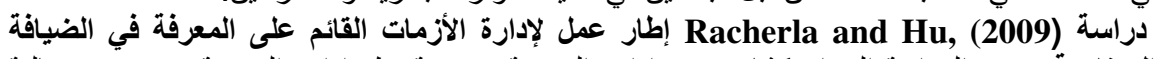

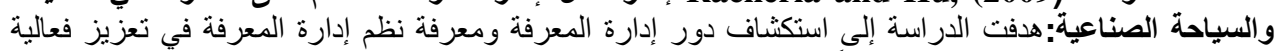

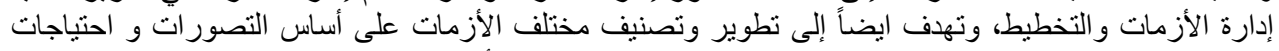

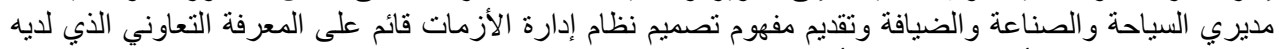

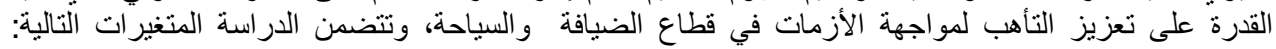




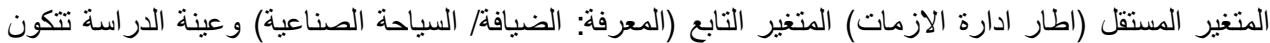

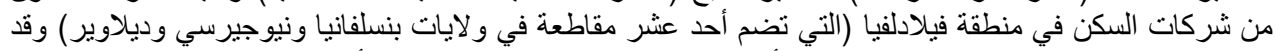

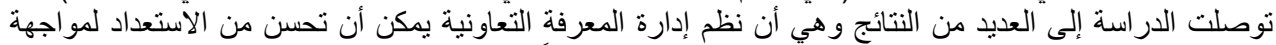

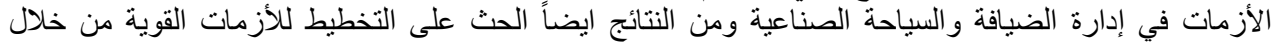
التحضير النشط و التدريب و التمارين.

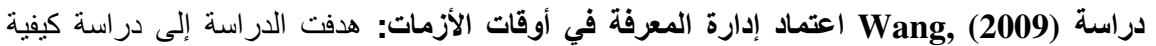

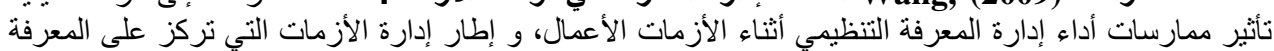

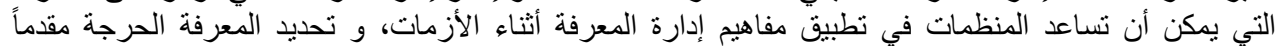

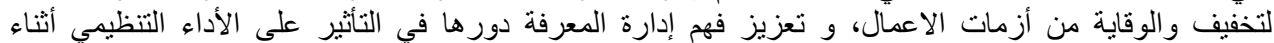

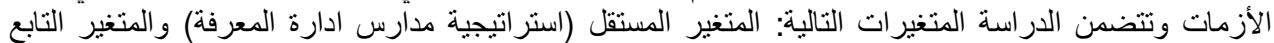

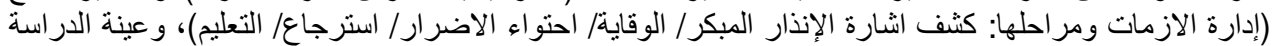

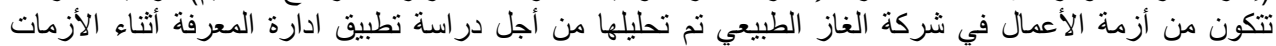

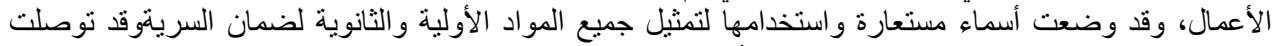

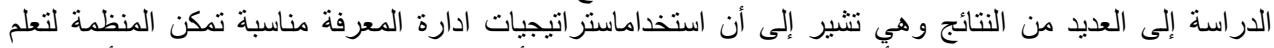

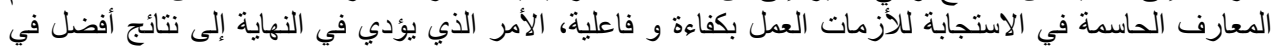

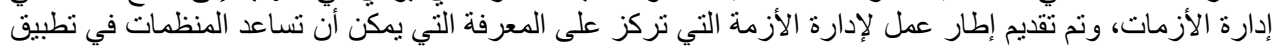
مفاهيم ادارة المعرفة أثناء الأزمات.

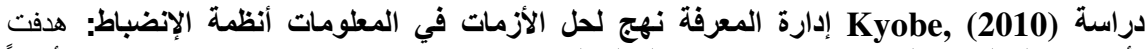

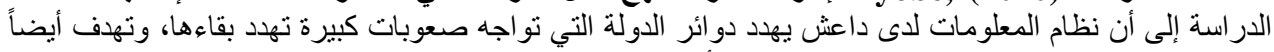

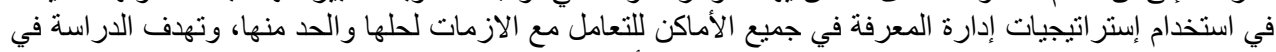

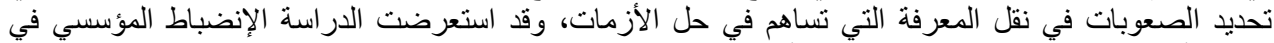

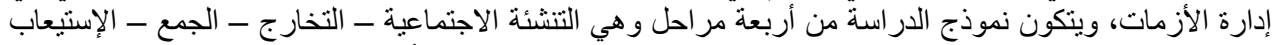

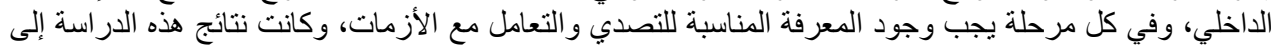

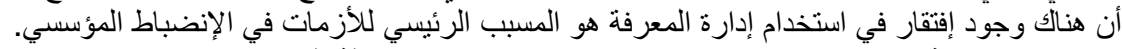

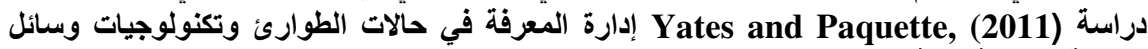

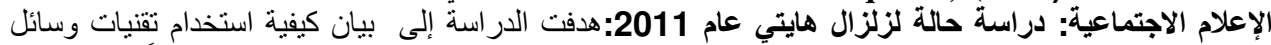

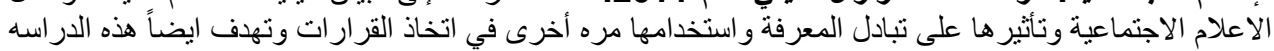

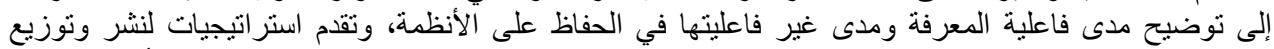

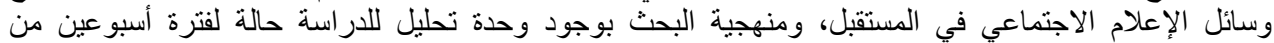

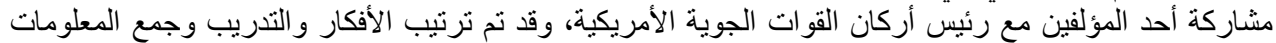

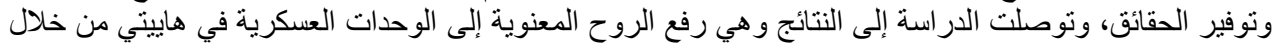

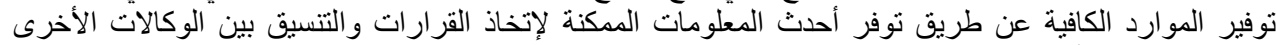

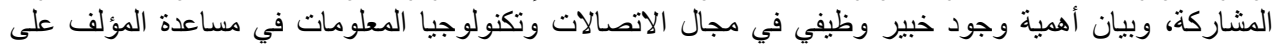

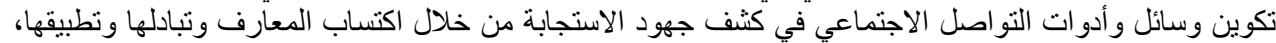

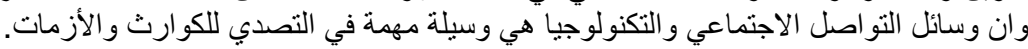

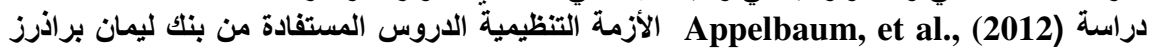

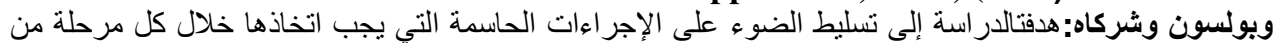

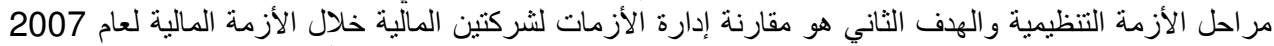

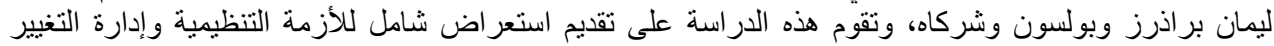

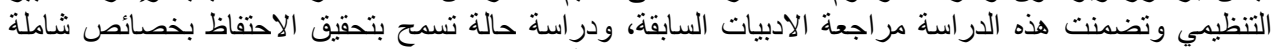

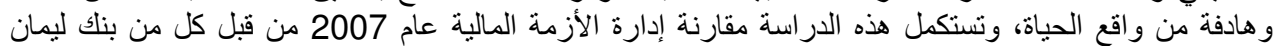

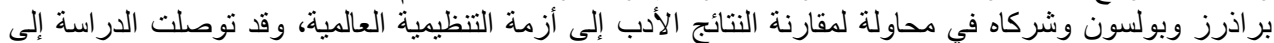

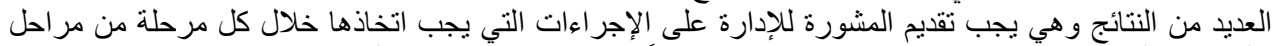

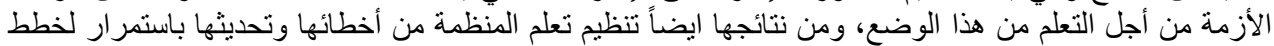

دراسة (2012) Jia, et al., إطار من نظم إدارة المعرفة لإدارة الأزمات السياحية:هدفت الدراسة إلى الإسلى إدارة الأزمات الخاصة بهم التعنة

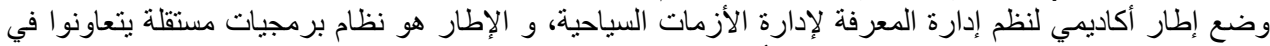

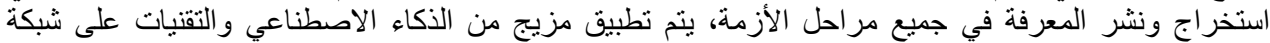

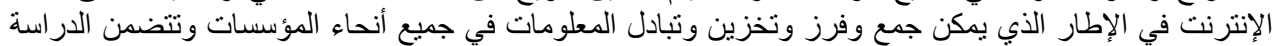

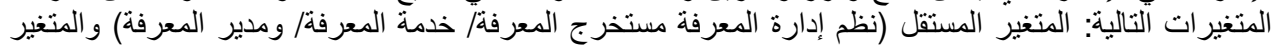




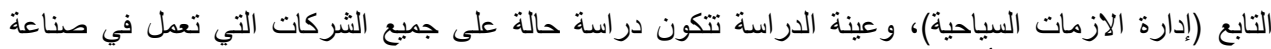

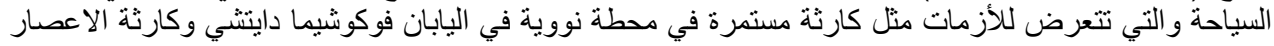

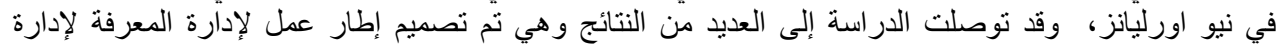

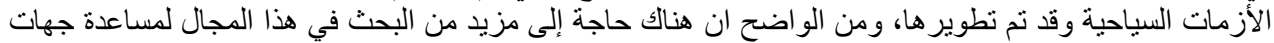

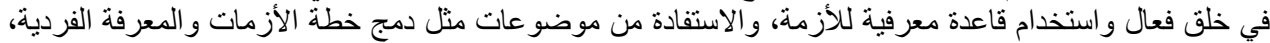

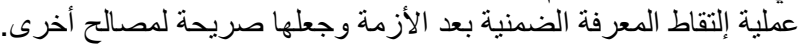

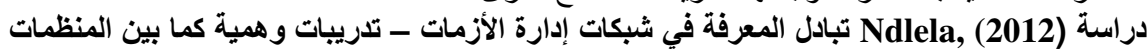

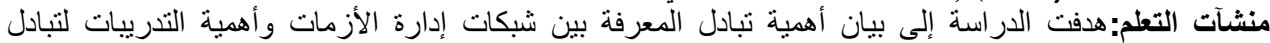

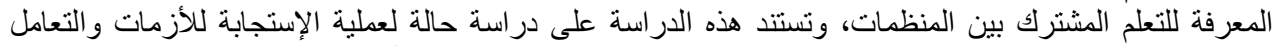

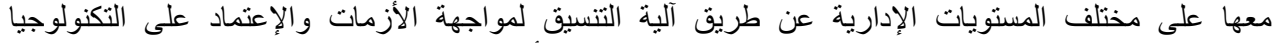

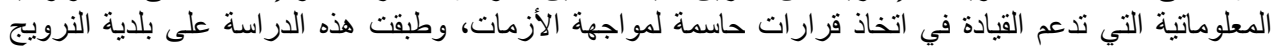

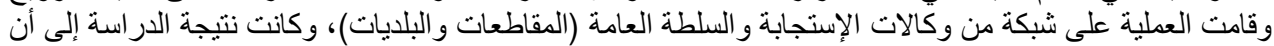

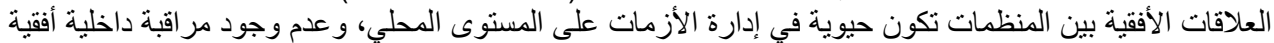

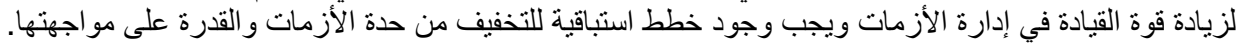

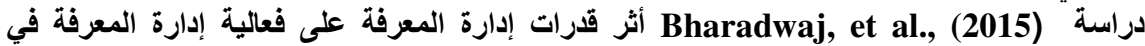

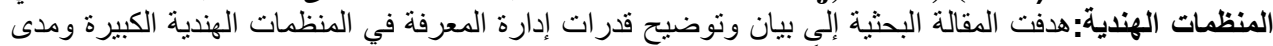

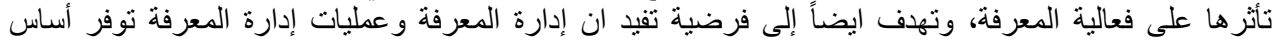

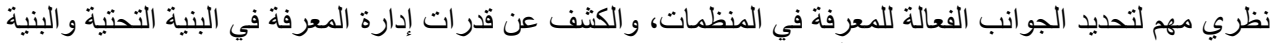

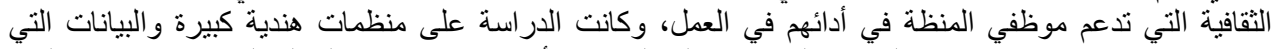

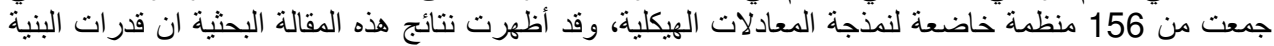

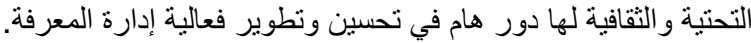

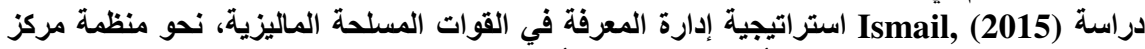

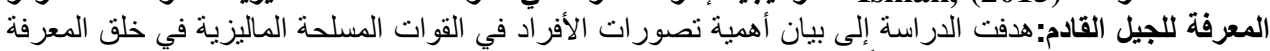

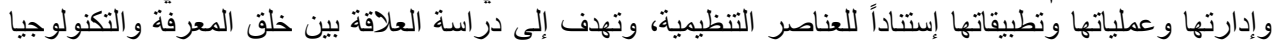

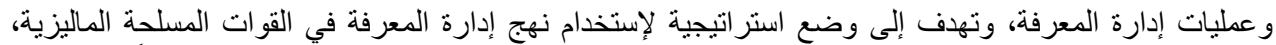

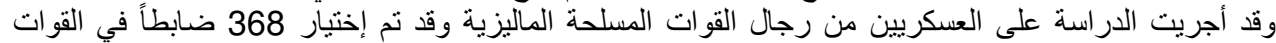

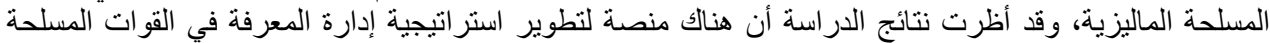

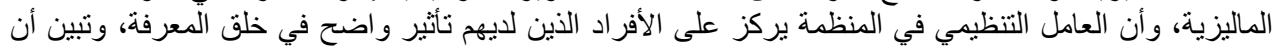

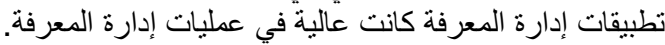

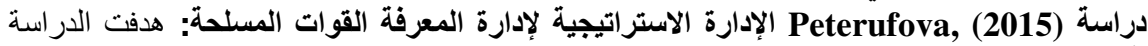

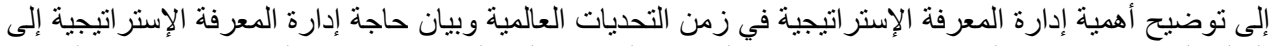

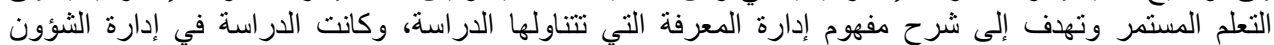

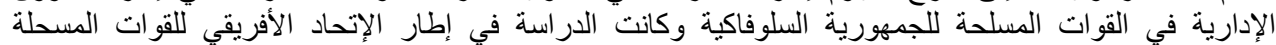

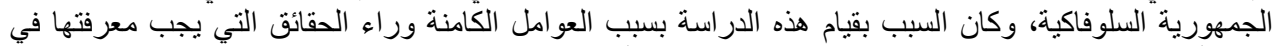

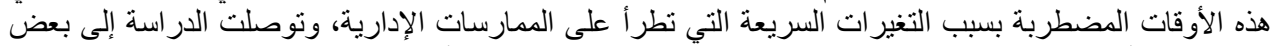

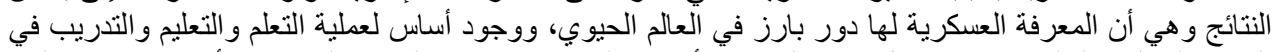

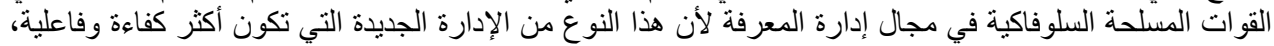

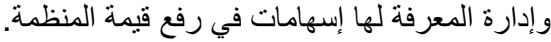

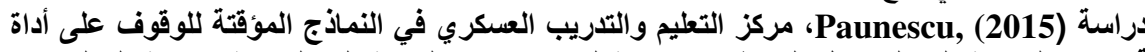

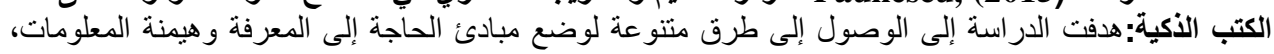

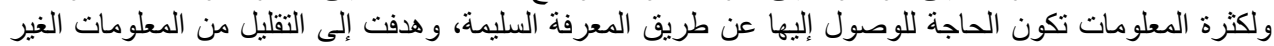

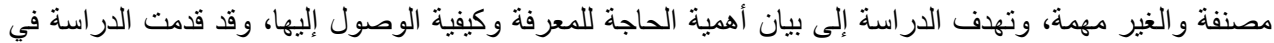

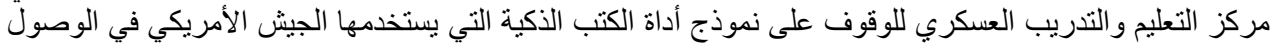

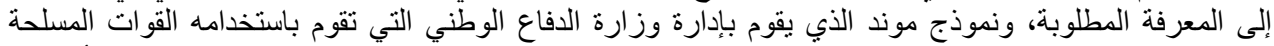

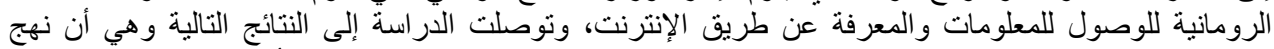

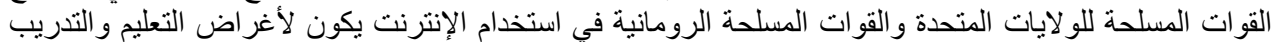

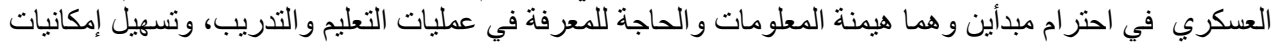

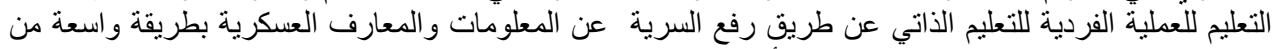

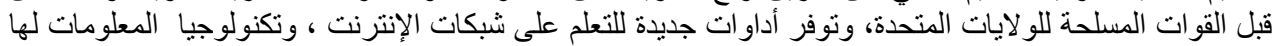


أثر كبير على الطريقة التي نستطيع بها فهم المكان و الزمان في عملية صنع القرار، وأهمية وجود مر اجع مفتوحة

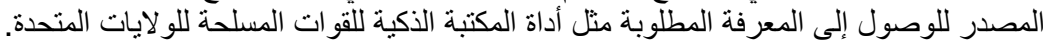

الخاتمة

من خلال عرض الدراسات السابقة في أثر إدارة المعرفة على إدارة الأزمات التنظيمية، نجد العديد من الدراسات

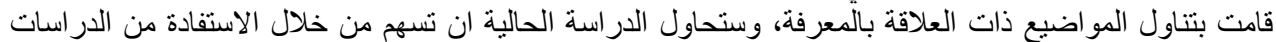

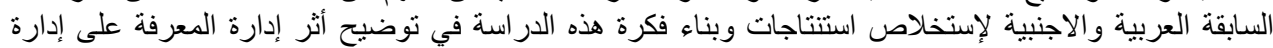

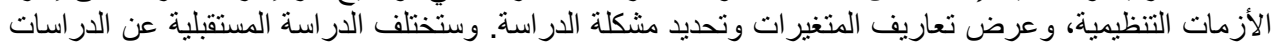

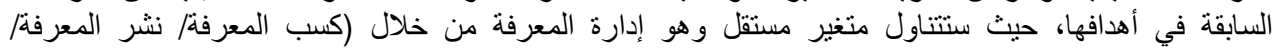

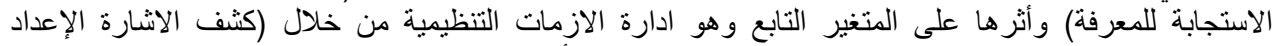

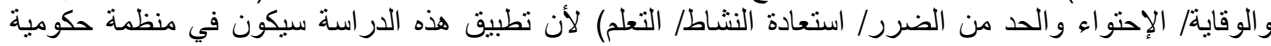

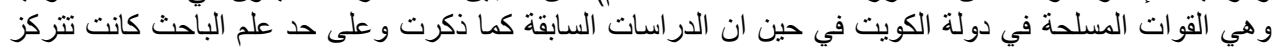

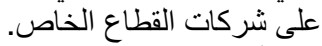

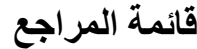

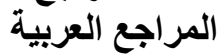

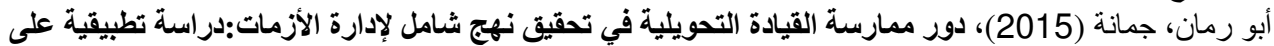

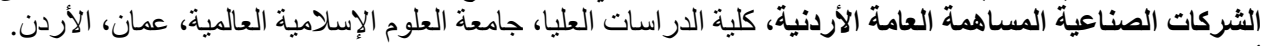

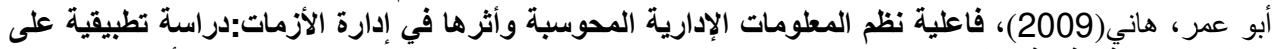

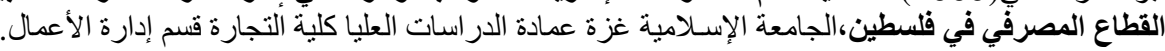

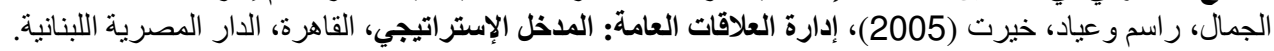

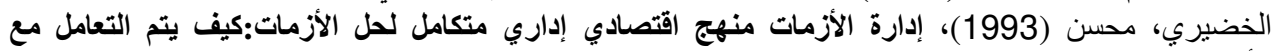

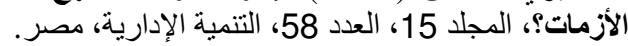

الخضيري، محسن (2002)، إدارة الأزمات: علم امتلاك كامل القوة في أثد لحظات الضعف، القاهرة، مجموعة النيل

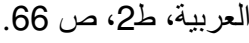
الديب، إبر اهيم (2008)، بحث في إدارة المعرفة، الأكاديمية العربية المفتوحة، الدنمارك، كلية الإدارة والإقتصاد.

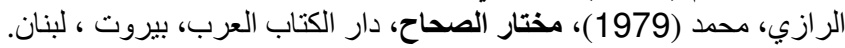

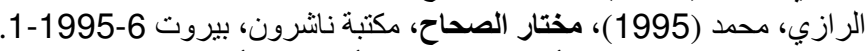

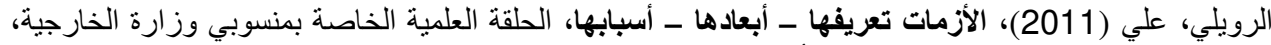
كلية التدريب، جامعة نايف العربية للعلوم الأمنية.

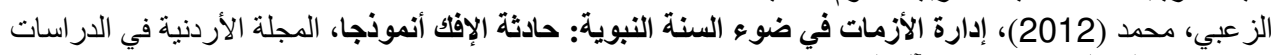
الإسلامية، كلية الثريعة، جامعة آل البيت.

السهلي، مثبب (2014)، فاعلية الثقافة التنظيمية في إدارة الازمات الأمنية: دراسة تطبيقية بجهاز الحرس الوطنية الوطني

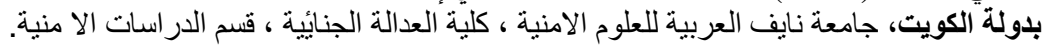

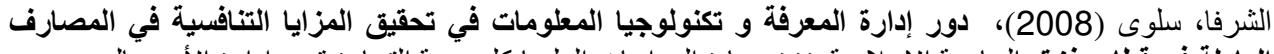

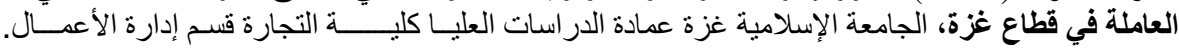

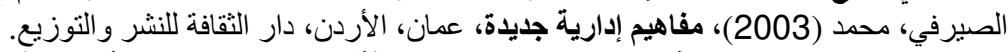

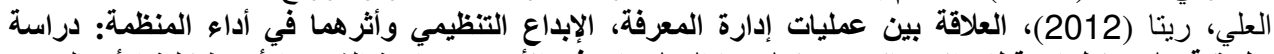

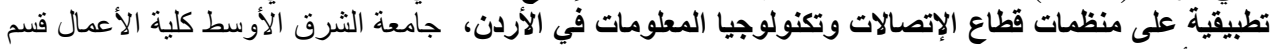

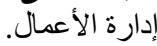

العلي، عبدالستار، قنديلجي، عامر والعمري، غسان (2009)، المدخل إلى إدارة المعرفة، الطبعة الثالثة، دار

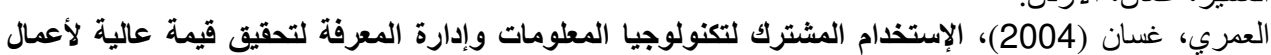

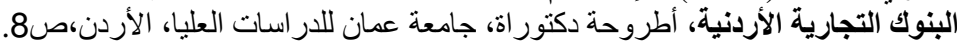

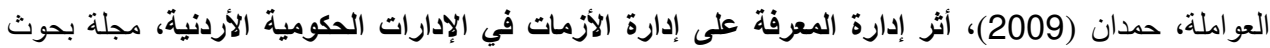

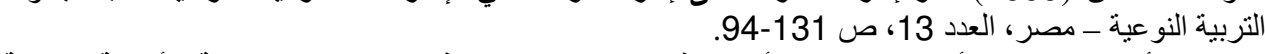
القطاونة، أبمن (2012)، أثثر نظام إدارة الأزمات في المناخ التئنة التظيمي في المصارف التجارية الأردنية: دراسة

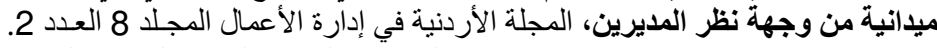
الكبيسي، صلاح الدين (2005)، إدارة المعرفة، المنظمة العربية للتنمية الإدارية، القاهرة، مصر ، صل صلإن 73. 
المطيران، مطيران (2007)، إدارة نظم المعرفة، المؤتمر الدولي حول الجودة الثاملة في ظل إدارة المعرفة

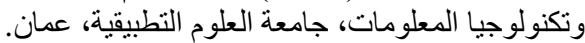

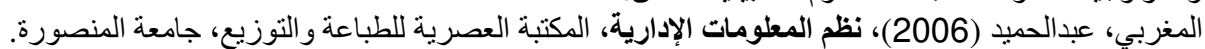

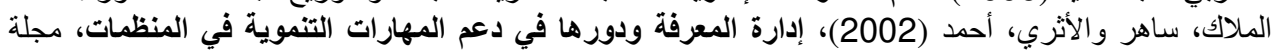

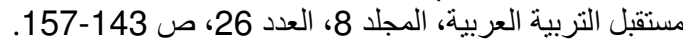

الملكاوي، إبر اهيم (2007)، إدارة المعرفة: الممارسات والمفاهيم، مؤسسة الوراق للنشر والتوزيع، الطبعة 1، صـ ص 308-1

الهريش، ثامر (2009)، إدارة المعرفة وإمكانية تطبيقها في دوريات الأمن العام بمدينة الرياض، جامعة نايف

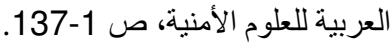

باسردة، توفيق (2008)، تكامل الدارة المبرة المعرفة والجودة الثاملة واثره في الاداء: دراسة تطبيقية في شركات

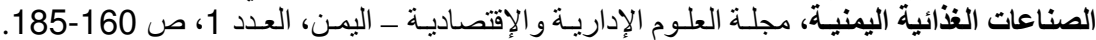

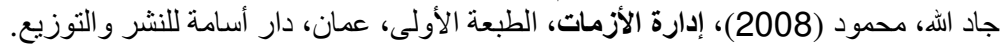

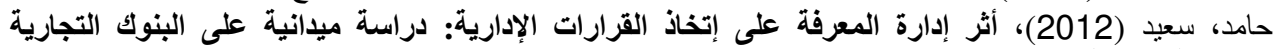

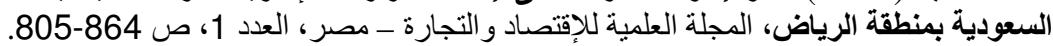

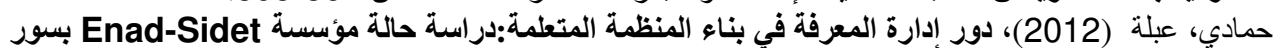

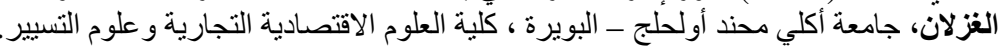

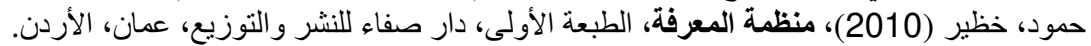

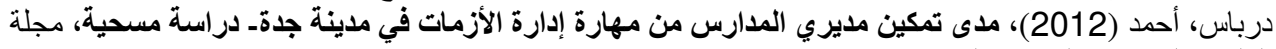
العلوم و الثقافة، مجلد 12، السون السودان.

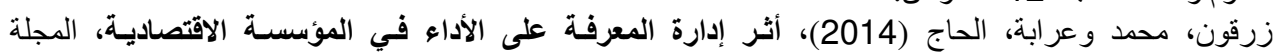

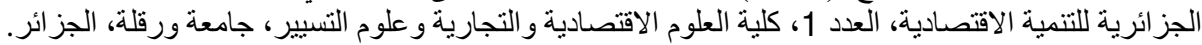

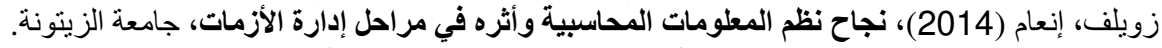

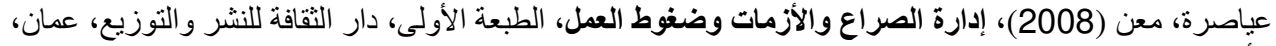

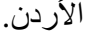
ماهر، أحمد (2006)، إدارة الأزمات، الدار الجامعية، الإسكندرية، مصر.

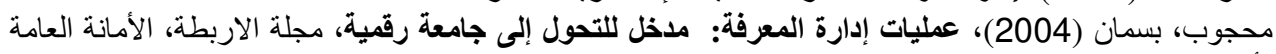

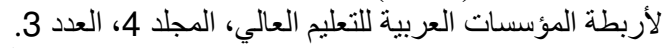

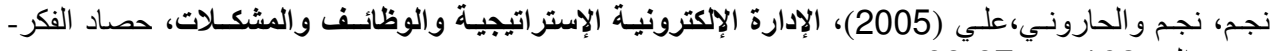

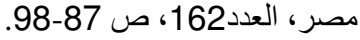

همشري، عمر (2013)، إدارة المعرفة الطريق إلى التميز والريادة، دار صفاء للطباعة والنشر والتوزيع، الطبعة 1، مجلدات 1. - مبري،

\section{References}

Altamony, H., Alshurideh, M., \& Obeidat, B. (2012). Information Systems for Competitive Advantage: Implementation of an Organizational Strategic Management Process. Proceedings of the 18th IBIMA Conference on Innovation and Sustainable Economic Competitive Advantage: From Regional Development to World Economic, Istanbul, Turkey, 9th-10th May.

Anbumathi, R., \& Sivasubramanian, R. (2016). Amalgamation of Talent and Knowledge Management Practices: A Conceptual Research Model. Gurgaon, United Kingdom, 20(4), 278-289.

Appelbaum, S., Keller, S., Alvarez, H., \& Bédard, C. (2012). Organizational Crisis: Lessons from Lehman Brothers and Paulson \& Company. International Journal of Commerce and Management, 22(4), 286-305. 
Backman, T. (2001). Creating Business Value from Knowledge Management, In Ramon C. Barquin (Ed), Knowledge Management. Verginia, Management Concept Vienna, 54.

Barry, B., \& Frank, B. (2013). Knowledge Management in Defence, North Atlantic Treaty Organization-NATO. European Conference on Knowledge Management, 106-116.

Bharadwaj, S, Chauhan, S., \& Raman, A, (2015). Impact of Knowledge Management Capabilities on Knowledge Management Effectiveness in Indian Organizations. Indian Institute of Management, Ahmedabad, The Journal for Decision Makers, 40(4), 421-434.

Duffy, J. (2000). Knowledge Management: To Be or Not to Be?, ARMA International, Information Management Journal Publisher, 34(1), 1535-2897.

Evangelista, P., Esposito E., Lauro V., \& Raffa, M. (2010). The Adoption of Knowledge Management Systems in Small Firms. Electronic Journal of Knowledge Management, 8(1), 33-42.

Falkheimer, J., \& Heide, M. (2006). Multicultural Crisis Communication: Towards a Social Constructionist Perspective. Journal of Contingencies \& Crisis Management, 14(4), 180-189.

Fearnley, R., \& Horder, M. (1997). What is Knowledge Management? Knowledge Management in the Oil and Gas Industry, London Conference Proceedings Notes.

Hajir, J., Obeidat, B., \& Al-dalahmeh, M. (2015). The Role of Knowledge Management Infrastructure in Enhancing Innovation at Mobile Telecommunication Companies in Jordan. European Journal of Social Sciences, 50(3), 313-330.

Holsapple, C., \& Singh, M. (2001). The Knowledge Value Chain Model: Activities for Competitiviveness. International Handbooks on Information Systems, 20(1), 215-251.

Hutchins, H., \& Wang, J. (2008). Organizational Crisis Management and Human Resource Development: A Review of the Literature and Implications to HRD Research and Practice. Advances in Developing Human Resources, 10(3).

Ismail, M. (2015). Knowledge Management Strategy in The Malaysian Armed Forces, Towards Next Gentric Organization. The Journal of Defence and Security, Kuala Lumpur, Science \& Technology Research Institute for Defence (STRIDE), 5(2), 216.

Jia, Z., Shi, Y., Jia, Y., \& Li, D. (2012). A Framework of Knowledge Management Systems for Tourism Crisis Management. International Workshop on Information and Electronics Engineering, 29, 138-143.

Jyoti, J., Gupta, P., \& Kotwal, S. (2011). Impact of Knowledge Management Practices on Innovative Capacity: A Study of Telecommunication Sector. Sage Journals, 15(4), 315330 .

Kateb, M., Swies, R., Obeidat, B., \& Maqableh, M. (2015). An Investigation on the Critical Factors of Information System Implementation in Jordanian Information Technology Companies. European Journal of Business and Management, 7(36), 11-28. 
Kyobe, M. (2010). A Knowledge Management Approach to Resolving the Crises in the Information Systems Discipline. Journal of Systems and Information Technology, 12(3), 161-173.

Peacock, M., Carson, B., \& Marquardt, M. (2015). Action Learning is and Leadership Development. Academy of Human Resource Development, 18(3).

Masa'deh, R. (2012). The Impact of Management Information Systems (MIS) on Quality Assurance (QA): A Case Study in Jordan. International Journal of Information, Business, and Management, 93-110.

Masa'deh, R. (2013). The Impact of Information Technology Infrastructure Flexibility on Firm Performance: An Empirical Study of Jordanian Public Shareholding Firms. Jordan Journal of Business Administration, 204-224.

Masa'deh, R., \& Shannak, R. (2012). Intermediary Effects of Knowledge Management Strategy and Learning Orientation on Strategic Alignment and Firm Performance.

Research Journal of International Studies, 112-128.

Masa'deh, R., Gharaibeh, A., Maqableh, M., \& Karajeh, H. (2013). An Empirical Study of Antecedents and Outcomes of Knowledge Sharing Capability in Jordanian Telecommunication Firms: A Structural Equation Modeling Approach. Life Science Journal, 10(4), 2284-2296.

Masa'deh, R., Gharaibeh, A., Tarhini, A., \& Obeidat, O. (2015). Knowledge Sharing Capability: A Literature Review. In Fourth Scientific \& Research Conference on New Trends in Business, Management and Social Sciences, Istanbul, Turkey, 19-20 September 2015, 1-16.

Masa'deh, R., Hunaiti, Z., \& Bani Yaseen, A. (2008). An Integrative Model Linking ITBusiness Strategic Alignment and Firm Performance: The Mediating Role of Pursuing Innovation and Knowledge Management Strategies. Communications of the International Business Information Management Association (IBIMA) Journal, 2(24), 180-187.

Masa'deh, R., Maqableh, M., \& Karajeh, H. (2014). A Theoretical Perspective on the Relationship between Leadership Development, Knowledge Management Capability, and Firm Performance. Asian Social Science, 10(6), 128.

Masa'deh, R., Shannak, R., Maqableh, M., \& Tarhini, A. (2017). The Impact of Knowledge Management on Job Performance in Higher Education: The Case of the University of Jordan. Journal of Enterprise Information Management, 30(2), 244-262.

Massingham, P. (2014). An Evaluation of Knowledge Management Tools: Part 1Managing Knowledge Resources. Journal of Knowledge Management, 18(6), 1075-1100.

Ndlela, M. (2012). Knowledge Sharing in Crisis Management Networks- Mock Drills as Inter-Organizational Learning Platforms, Hedmark University College, Norway.

Obeidat, B., Al-Suradi, M., Masa'deh, R. \& Tarhini, R. (2016). The Impact of Knowledge Management on Innovation. Management Research Review, 39(10), 1214-1238. 
Oxford, (1994). Paperback Dictionary, Oxford University Press.

Paraskevas, A. (2006). Crisis management or Crisis Response System?, A Complexity Science Approach to Organizational Crises. Management Decision, 44(7), 892-907.

Patrides, L. (2002). Organizational Learning and the Case for Knowledge Based Systems, 2002(113), 84-69.

Paunescu, M. (2015). The Miltary Education and Training Posture in The Contemporary Paradigms of Warfare the Smart Book's. Journal Conference proceedings of eLearning and Software for Education (eLSE), 1, 476-482.

Peterufova, M. (2015). Strategic Knowledge Management in The Armed Forces: Of General M. R. Stefanik in Liptovsky Mikulas. Journal of Defense Resources Management, 2, 51-56.

Racherla, P., \& Hu, C. (2009). A Framework for Knowledge Based Crisis Management in the Hospitality, 50(4), 561-577.

Schwandt, D., \& Marquardt, J. (2000). Organizational Learning: From World-Class Theories to Global Best Practices, Library of Congress Classification, 258.

Shannak, R., Obeidat, B., \& Almajali, D. (2010). Information Technology Investments: A Literature Review. Proceedings of the 14th IBIMA Conference on Global Business Transformation through Innovation and Knowledge Management: An Academic Perspective, Istanbul-Turkey, 23rd-24th June, 1356-1368.

Shaw, D., Hall, M., Edwards, J., \& Baker, B. (2007). Responding to Crisis through Strategic Knowledge Management. Journal of Organizational Change Management, 20(4), $559-578$.

Valmohammadi, C., \& Ahmadi, M. (2015). The Impact of Knowledge Management Practices on Organizational Performance: A Balanced Scorecard Approach. Journal of Enterprise Information Management, 28(1), 131-159.

Vratskikh, I., Al-Lozi, M., \& Maqableh, M. (2016). The Impact of Emotional Intelligence on Job Performance via the Mediating Role of Job Satisfaction. International Journal of Business and Management, 11(2), 69-91.

Wang, W. (2009). Knowledge Management Adoption in Times of Crisis. Industrial Management \& Data Systems, 109(4), 445-462.

Yates, D., \& Paquette, S. (2011). Emergency Knowledge Management and Social Media Technologies: A Case Study of the 2010 Haitian Earthquake. International Journal of Information Management, 31(1), 6-13. 